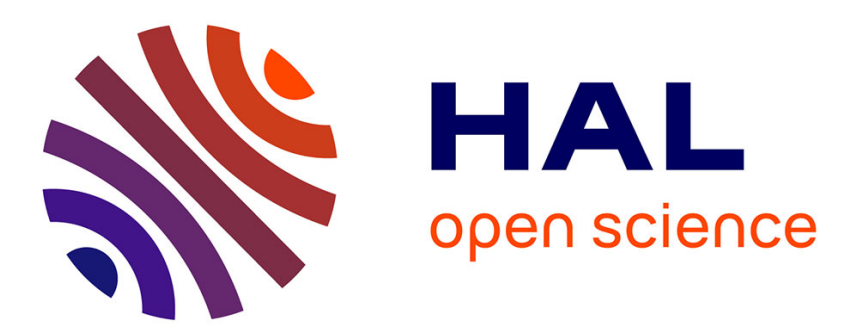

\title{
A high-order density-based finite volume method for the computation of all-speed flows
}

\author{
Xesús Nogueira, Luis Ramírez, Sofiane Khelladi, Jean-Camille Chassaing, \\ Ignasi Colominas
}

\section{- To cite this version:}

Xesús Nogueira, Luis Ramírez, Sofiane Khelladi, Jean-Camille Chassaing, Ignasi Colominas. A highorder density-based finite volume method for the computation of all-speed flows. Computer Methods in Applied Mechanics and Engineering, 2016, 298, pp.229-251. 10.1016/j.cma.2015.10.004 . hal02446726

\section{HAL Id: hal-02446726 \\ https://hal.science/hal-02446726}

Submitted on 21 Jan 2020

HAL is a multi-disciplinary open access archive for the deposit and dissemination of scientific research documents, whether they are published or not. The documents may come from teaching and research institutions in France or abroad, or from public or private research centers.
L'archive ouverte pluridisciplinaire HAL, est destinée au dépôt et à la diffusion de documents scientifiques de niveau recherche, publiés ou non, émanant des établissements d'enseignement et de recherche français ou étrangers, des laboratoires publics ou privés. 


\title{
A high-order density-based finite volume method for the computation of all-speed flows
}

\author{
Xesús Nogueira ${ }^{\mathrm{a}, *}$, Luis Ramírez ${ }^{\mathrm{a}}$, Sofiane Khelladi ${ }^{\mathrm{b}}$, Jean-Camille \\ Chassaing $^{\mathrm{c}}$, Ignasi Colominas ${ }^{\mathrm{a}}$ \\ ${ }^{a}$ Group of Numerical Methods in Engineering, Universidade da Coruña, Campus de \\ Elviña, 15071, A Coruña, Spain \\ ${ }^{b}$ Arts et Métiers ParisTech, 151 boulevard de l'Hôpital, F-75013 Paris, France \\ ${ }^{c}$ Sorbonne Universités, UPMC Univ Paris 06, CNRS, UMR 7190, Institut Jean Le Rond \\ d'Alembert, F-75005 Paris, France
}

\begin{abstract}
In this paper we present a high-order density-based finite-volume framework for all-speed flows. The formulation is based on high-order variable reconstructions performed using Moving Least Squares approximations. In particular, we show that combining high-order discretization schemes with low-Mach fixes, it is possible to remove the grid dependency problem at low Mach numbers on both structured and unstructured grids. In order to maintain the accuracy and the robustness of the numerical method at transonic conditions, different procedures are proposed, based on the use of a selective limiting.

Keywords: High-order methods, low-Mach flows, Finite Volume, All-speed flows, Moving Least Squares
\end{abstract}

\footnotetext{
${ }^{*}$ Corresponding author, e-mail: xnogueira@udc.es
} 


\section{Introduction}

Traditionally, two families of finite volume schemes have been developed to compute either compressible or incompressible flows. Density-based solvers $[1,2,3]$ are used for the computation of flows when compressibility effects are important (mainly transonic, supersonic and hypersonic flows), whereas pressure-based solvers $[4,5,6]$ are designed to compute incompressible flows. In both techniques, the velocity is obtained from the momentum equations. The difference between the two approaches is the computation of the pressure field. In density-based solvers, the density is computed from the continuity equation and then the pressure is obtained from an equation of state. Pressure-based solvers compute the pressure by solving a Poisson-type equation, obtained from continuity and momentum equations.

In pressure-based solvers, the SIMPLE and related algorithms [5] have been widely used to compute incompressible flows, and several authors have developed methods to extend these algorithms to all-speed flows [7, 8].

From a practical point of view, density-based schemes are not suitable for flows with Mach number lower than 0.3 [9]. These solvers present a number of problems: stiffness of the equations, cancellation in the pressure variable [10] and the loss of accuracy due to an excessive numerical diffusion (accuracy problem).

In some cases, resorting to solvers dealing with all-speed flows cannot be avoided because of the importance of flows where low and high Mach regions are present (for example flow past an aerodynamic profile at high angle of attack, or flow past a blunt body), or when compressibility effects are important, even in low Mach number flows. Thus, the modification of density 
or pressure-based solvers to compute all-speed flows is a current active area of research. It is known that the accuracy problem of density-based solvers in low-Mach flows is originated by the introduction of spurious pressure and velocity waves that avoid the velocity field to verify (or at least be close to) the zero-divergence constraint $[11,12,13,14,15]$. Preconditioning techniques $[12,13,16,17,18]$ have been developed to increase the accuracy (and also to overcome the stifness problem) of Godunov schemes in low Mach flows. The preconditioning matrix multiplies the time derivatives of the set of equations, with the effect of re-scaling the eigenvalues (acoustic-speed) of the system, but paying the price of spoiling the temporal accuracy of the scheme. Thus, this technique was initially developed for steady flows, but extensions to unsteady flow have been proposed by using a dual time stepping technique [19, 20]. An Asymptotic-Preserving methodology has been presented in [21].

In [22] a correction for the numerical dissipation of the Roe's approximate Riemann solver was introduced. This correction allows using Roe's flux for low Mach flows, and it was generalized to all-speed flows in [23]. In [24] it is stated that the fix to the accuracy problem is related to cancellation of the normal velocity jump. Related with this finding, it was reported [25] that the accuracy problem is not observed when the Roe's approximate Riemann solver is used in a first-order finite volume scheme on triangular grids. Based on these findings, some fixes for the numerical flux of Roe are presented in $[26,27,28]$. Other fixes have also been proposed for Roe flux and for other Riemann solvers, such as the HLL-family [29]. Several flux-splitting type schemes accurate at low and also high Mach have been presented in $[30,31,32]$. On the other hand, some authors have reported Discontinuous 
Galerkin (DG) solutions of low-Mach flow with and without preconditioning $[33,34,35]$. The use of high-order discretization schemes reduces the jumps in the normal velocity component alleviating the accuracy problem. It has been shown, however, that the accuracy problem is not completely solved [35], since a grid dependency with the Mach number still remains.

In this paper we present a high-order density-based finite-volume formulation for all-speed flows. The high-order numerical discretization and the selective limiting procedure are based on Moving Least Squares approximations $[36,37,38,39,40]$.

The outline of this study is as follows. The general formulation of the proposed all-speed scheme is presented in section 2. Then, the accuracy of

the numerical scheme is investigated in section 3. In section 4 we highlight the problem of using slope limiters with low-Mach fixes, and we present several procedures to solve this problem. The robustness and accuracy of the present all-speed formulation is assessed in section 5 by the computation of an unsteady transonic viscous flow over a circular cylinder. Finally, conclusions are drawn in section 6.

\section{General formulation}

In this work, we used a finite volume numerical framework that allows us to reach convergence orders in space greater than two [36, 38, 39]. This formulation is based on the use of Moving Least Squares [41, 42] for the computation of the derivatives required for the Taylor expansion in the reconstruction step of a Godunov-like method. The fundamentals of this method are exposed in the next section. 


\subsection{Higher-order Finite volume schemes based on Moving Least Squares}

The Navier-Stokes equations, written in a general form as a system of conservation laws, read

$$
\frac{\partial \boldsymbol{u}}{\partial t}+\nabla \cdot\left(\mathcal{F}^{H}+\mathcal{F}^{E}\right)=S \quad \text { in } \quad \Omega
$$

where $\boldsymbol{u}$ is the vector of variables, $\mathcal{F}^{H}$ is the inviscid flux vector, $\mathcal{F}^{E}$ is the viscous flux vector and $S$ is a source term. The set of equations needs to be supplemented with suitable initial and boundary conditions. These are a crucial point in low-Mach computations using the compressible system of equations. It has been shown $[12,14,43]$ that a set of "well-prepared" initial conditions is required for the convergence of the solution of the compressible system of equations to the solution of the incompressible set when $M \rightarrow 0$. In this context, "well-prepared" means that the initial pressure field scales with the square of the Mach number and that the initial velocity field is close to a divergence free field [12]. In addition, Dirichlet boundary conditions may also lead to inaccurate results of low Mach finite volume schemes [14].

The fluxes have been generically split into a hyperbolic-like part, $\mathcal{F}^{H}$, and an elliptic-like part, $\mathcal{F}^{E}$, that is null for the Euler equations. Consider, in addition, a partition of the domain $\Omega$ into a set of non-overlapping control volumes or cells, $\mathcal{T}^{h}=I$. Furthermore, we define a reference point (node), $\boldsymbol{x}_{I}$ inside each cell (the cell centroid). The spatial representation of a variable using MLS is explained in the following. Let us consider a function $\boldsymbol{u}(\boldsymbol{x})$, given by its point values, $\boldsymbol{u}_{I}=\boldsymbol{u}\left(\boldsymbol{x}_{I}\right)$, at the cell centroids, with coordinates $x_{I}$.

We write the approximation $\boldsymbol{u}^{h}(\boldsymbol{x})$ in terms of a set of shape functions 
$\left\{N_{I}(\boldsymbol{x})\right\}$ associated to the nodes, such that $\boldsymbol{u}^{h}(\boldsymbol{x})$ is given by

$$
\boldsymbol{u}^{h}(\boldsymbol{x})=\sum_{j=1}^{n_{I}} N_{j}(\boldsymbol{x}) \boldsymbol{u}_{j}
$$

which states that the approximation at a given position $\boldsymbol{x}$ is computed using certain $n_{I}$ neighboring nodes. This set of nodes is referred to as the stencil associated to the evaluation point $\boldsymbol{x}$. This is schematically shown in Figure 1. The set of basis functions $\boldsymbol{N}(\boldsymbol{x})$ is computed using the Moving Least Squares method. The gradient can be computed as follows

$$
\boldsymbol{\nabla} \boldsymbol{u}^{h}(\boldsymbol{x})=\sum_{j=1}^{n_{I}} \boldsymbol{\nabla} N_{j}(\boldsymbol{x}) \boldsymbol{u}_{j}
$$

and high-order derivatives can be computed using the same rationale. We refer the interested reader to $[36,38,39]$ for a complete description of the computation of the MLS shape functions and its derivatives.

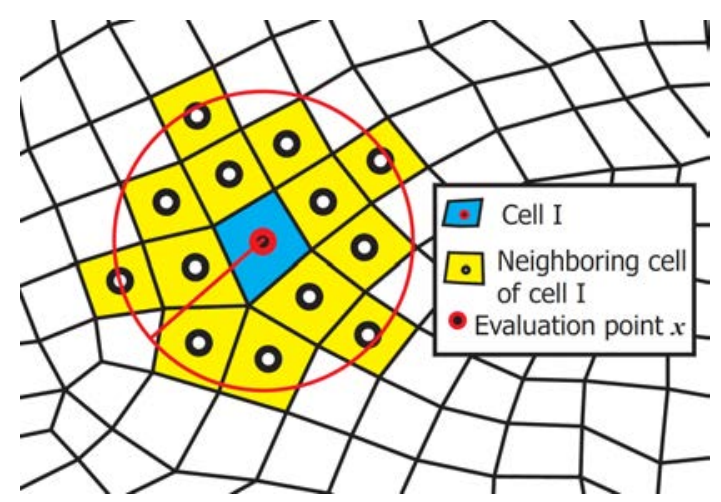

Figure 1: Scheme of the stencil of a reference control volume

Note that MLS approximations have a centered character. We also note that, using MLS, the approximate function $\boldsymbol{u}^{h}(\boldsymbol{x})$ is not a polynomial in general. The value of MLS shape functions at a point depends on the number 
of neighbors considered for this point $\left(n_{I}\right)$, a kernel function and a polynomial basis $[36,38]$. The function of the kernel is to weight the importance of each neighbor point in the approximation process. In this work we use a cubic polynomial basis and an exponential kernel, defined in 1D as

$$
W\left(x_{j}, x_{I}, s_{x}\right)=\frac{e^{-\left(\frac{d}{c}\right)^{2}}-e^{-\left(\frac{d m}{c}\right)^{2}}}{1-e^{-\left(\frac{d m}{c}\right)^{2}}}
$$

where $d=\left|x_{j}-x_{I}\right|, d_{m}=2 \max \left(\left|x_{j}-x_{I}\right|\right)$, with $j=1, \ldots, n_{I}, c=\frac{d_{m}}{s_{x}}, x_{I}$ is the position of a reference point, $x_{j}$ is the position of every cell centroid of the stencil and $s_{x}$ is a shape parameter, which in the present work is set to $s_{x}=3$.

A $n$-dimensional kernel can be obtained by multiplying $n$ 1D kernels. Thus, the $2 \mathrm{D}$ exponential kernel is the following

$$
W_{j}\left(\boldsymbol{x}_{j}, \boldsymbol{x}_{I}, s_{x}, s_{y}\right)=W_{j}\left(x_{j}, x_{I}, s_{x}\right) W_{j}\left(y_{j}, y_{I}, s_{y}\right)
$$

The integral form of the system of conservation laws (1) for each control volume $I$ is

$$
\int_{\Omega_{I}} \frac{\partial \boldsymbol{u}}{\partial t} d \Omega+\int_{\Gamma_{I}}\left(\mathcal{F}^{H}+\mathcal{F}^{E}\right) \cdot \boldsymbol{n} d \Gamma=\int_{\Omega_{I}} \boldsymbol{S} d \Omega
$$

where $\Omega_{I}$ is the control volume area, $\Gamma_{I}$ is the control volume perimeter and $\boldsymbol{n}=\left(n_{x}, n_{y}\right)^{T}$ is the unitary exterior normal of the contour.

Introducing the component-wise reconstructed function $\boldsymbol{u}^{h}$ we obtain

$$
\int_{\Omega_{I}} \frac{\partial \boldsymbol{u}^{h}}{\partial t} d \Omega+\int_{\Gamma_{I}}\left(\mathcal{F}^{h H}+\mathcal{F}^{h E}\right) \cdot \boldsymbol{n} d \Gamma=\int_{\Omega_{I}} \boldsymbol{S}\left(\boldsymbol{u}^{h}\right) d \Omega
$$

For hyperbolic problems, we introduce a "broken" reconstruction, $\boldsymbol{u}_{I}^{h b}$, which approximates $\boldsymbol{u}^{h}(\boldsymbol{x})$ (and, therefore, $\boldsymbol{u}(\boldsymbol{x})$ ) locally inside each cell $I$, 
and is discontinuous across cell interfaces [36]. This procedure allows us to compute the numerical flux at Gauss points at the interfaces. Thus, we can easily make use of Riemann solvers.

In general, we require the order of accuracy of the broken reconstruction to be the same as that of the original continuous reconstruction. Thus, using Taylor series expansions; a quadratic reconstruction inside cell $I$, reads

$$
\boldsymbol{u}_{I}^{h b}(\boldsymbol{x})=\boldsymbol{u}_{I}^{h}+\nabla \boldsymbol{u}_{I}^{h} \cdot\left(\boldsymbol{x}-\boldsymbol{x}_{I}\right)+\frac{1}{2}\left(\boldsymbol{x}-\boldsymbol{x}_{I}\right)^{T} \boldsymbol{H}^{h}\left(\boldsymbol{x}-\boldsymbol{x}_{I}\right)
$$

where the gradient $\boldsymbol{\nabla} \boldsymbol{u}_{I}^{h}$ and the Hessian matrix $\boldsymbol{H}^{h}$ involve the successive derivatives of the continuous reconstruction $\boldsymbol{u}^{h}(\boldsymbol{x})$, which are evaluated at the cell centroids using MLS according to equation (3). This dual continuous/discontinuous reconstruction of the solution is crucial in order to obtain accurate and efficient numerical schemes for mixed parabolic/hyperbolic problems. The cell-wise broken reconstruction defined here is actually a piecewise continuous approximation to $\boldsymbol{u}^{h}$. The advantage is that it allows to make use of Riemann solvers, limiters, and other standard finite volume technologies, while keeping some consistency in terms of functional representation. Thus, considering the Navier-Stokes equations, the general continuous reconstruction is used to evaluate the viscous (elliptic-like) fluxes, whereas its discontinuous approximation is used to evaluate the inviscid (hyperbolic-like) fluxes.

The final semi-discrete scheme for the continuous/discontinuous approach can be written as 


$$
\int_{\Omega_{I}} \frac{\partial \boldsymbol{u}^{h}}{\partial t} d \Omega+\int_{\Gamma_{I}} \boldsymbol{\Theta}\left(\boldsymbol{u}^{h b+}, \boldsymbol{u}^{h b-}\right) d \Gamma+\int_{\Gamma_{I}} \mathcal{F}^{h E} \cdot \boldsymbol{n} d \Gamma=\int_{\Omega_{I}} \boldsymbol{S}\left(\boldsymbol{u}^{h}\right) d \Omega
$$

where $\boldsymbol{\Theta}\left(\boldsymbol{u}^{h b+}, \boldsymbol{u}^{h b-}\right)$ is a suitable numerical flux, and + and - refers to the left and right states of the cell $I$.

It is known that at low Mach numbers, the compressible Euler equations present a stiff behavior due to large differences in wave speeds. Several preconditioning techniques have been developed to alleviate this problem (see $[12,13,16,17,18]$ among others). Since such study is beyond the scope of this paper, we employed the third-order Runge-Kutta TVD scheme of Shu and Osher [44] as time-integration scheme.

\subsection{Numerical Flux strategy for all-speed flows}

In this paper, both Roe [1] and Rusanov [45] Riemann solvers are combined with higher-order reconstruction schemes to perform all-speed flow computations. Roe solver was selected due to the fact that it has been shown that first-order schemes based in Roe flux does not present the accuracy problem on triangular grids [25].

It is known that one of the advantages of Rusanov flux is its simple formulation, independently of the equation of state (EOS) used, in opposition to Roe flux, for instance, which presents a more complex implementation when the ideal gas EOS is changed.

However, the numerical entropy production is higher than for the Roe scheme, and thus, the computation of low Mach flows with this scheme presents more difficulties [46]. Moreover, the numerical dissipation of the Rusanov scheme depends on the Mach number $M$ in the form $o\left(\frac{M}{\Delta x}\right)$ as 
$M \rightarrow 0$, resulting in an asymptotically inconsistent scheme with respect to the Mach number.

\subsubsection{Roe Flux}

The Roe flux [1] can be written as

$$
\Theta_{i+\frac{1}{2}}=\frac{1}{2}\left(\mathcal{F}^{h H+}+\mathcal{F}^{h H-}\right) \cdot \boldsymbol{n}-\frac{1}{2} \sum_{k=1}^{4} \tilde{\alpha}_{k}\left|\tilde{\lambda}_{k}\right| \tilde{\boldsymbol{r}}_{k}
$$

where the variables corresponding to the left and right states are evaluated according to the Taylor series expansion (8) and MLS shape functions $(2,3)$.

In equation (10) $\tilde{\lambda}_{k}$ and $\tilde{\boldsymbol{r}}_{k}$ are the eigenvalues and eigenvectors of the approximated Jacobian [1] defined as

$$
\begin{gathered}
\tilde{\lambda}_{1}=\tilde{\boldsymbol{v}} \cdot \boldsymbol{n}-\tilde{c} \\
\tilde{\lambda}_{2}=\tilde{\lambda} \tilde{\lambda}_{3}=\tilde{\boldsymbol{v}} \cdot \boldsymbol{n} \\
\tilde{\lambda}_{4}=\tilde{\boldsymbol{v}} \cdot \boldsymbol{n}+\tilde{c} \\
{\left[\tilde{\boldsymbol{r}}_{1}, \tilde{\boldsymbol{r}}_{2}, \tilde{r}_{3}, \tilde{\boldsymbol{r}}_{4}\right]=\left(\begin{array}{cccc}
1 & 0 & 1 & 0 \\
\tilde{u}-\tilde{c} n_{x} & -\tilde{c} n_{y} & \tilde{u} & \tilde{u}+\tilde{c} n_{x} \\
\tilde{v}-\tilde{c} n_{y} & -\tilde{c} n_{x} & \tilde{v} & \tilde{v}+\tilde{c} n_{y} \\
\tilde{H}-\tilde{c} \tilde{\boldsymbol{v}} \cdot \boldsymbol{n} & \tilde{c}\left(\tilde{v} n_{x}-\tilde{u} n_{y}\right) & \frac{1}{2}\left(\tilde{u}^{2}+\tilde{v}^{2}\right) & \tilde{H}+\tilde{c} \tilde{\boldsymbol{v}} \cdot \boldsymbol{n}
\end{array}\right)}
\end{gathered}
$$

where $H$ is the enthalpy, $c$ is the sound velocity and $\tilde{\boldsymbol{v}}=(\tilde{u}, \tilde{v})^{T}$ is the velocity vector.

We also define $\tilde{\alpha}_{k}$ as

$$
\begin{aligned}
& \tilde{\alpha}_{1}=\frac{1}{2 \tilde{c}^{2}}\left[\Delta(p)-\tilde{\rho} \tilde{c}\left(\Delta(u) n_{x}+\Delta(v) n_{y}\right)\right] \\
& \tilde{\alpha}_{2}=\frac{\rho}{\tilde{c}}\left[\Delta(v) n_{x}-\Delta(u) n_{y}\right] \\
& \tilde{\alpha}_{3}=\frac{1}{\tilde{c}^{2}}\left[\Delta(p)-\tilde{c}^{2} \Delta(\rho)\right] \\
& \tilde{\alpha}_{4}=\frac{1}{2 \tilde{c}^{2}}\left[\Delta(p)+\tilde{\rho} \tilde{c}\left(\Delta(u) n_{x}+\Delta(v) n_{y}\right)\right]
\end{aligned}
$$


where symbol $\sim$ indicates Roe's average [1], and $\Delta(\cdot)=(\cdot)^{-}+(\cdot)^{+}$is computed using the high-order MLS reconstruction scheme depicted in section 2.1.

\subsubsection{Rieper's Fix for the Roe flux}

In this work, we use the low-Mach fix presented by Rieper in [27] as a generalization of the low-Mach $X$ schemes initially developed by Dellacherie [14]. It consists in a reduction of the normal velocity jump that is the term responsible of the accuracy problem. It is simply obtained by modifying the terms $\tilde{\alpha}_{1}$ and $\tilde{\alpha}_{4}$ in equation (13) as follows

$$
\begin{aligned}
& \tilde{\alpha}_{1}=\frac{1}{2 \tilde{c}^{2}}\left[\Delta(p)-\tilde{\rho} \tilde{c} f\left(M_{l}\right)\left(\Delta(u) n_{x}+\Delta(v) n_{y}\right)\right] \\
& \tilde{\alpha}_{4}=\frac{1}{2 \tilde{c}^{2}}\left[\Delta(p)+\tilde{\rho} \tilde{c} f\left(M_{l}\right)\left(\Delta(u) n_{x}+\Delta(v) n_{y}\right)\right]
\end{aligned}
$$

where $f\left(M_{l}\right)$ is a function of the local Mach number that is active when $M_{l}<1$. It is defined for a cell $I$ as

$$
f\left(M_{l}\right)=\min \left(M_{l}, 1\right)
$$

with

$$
M_{l}=\frac{|\tilde{u}|_{I}+|\tilde{v}|_{I}}{\tilde{c}_{I}}
$$

\subsubsection{Rusanov Flux}

The Rusanov flux [45] can be written as

$$
\boldsymbol{\Theta}_{i+\frac{1}{2}}=\frac{1}{2}\left(\mathcal{F}^{h H+}+\mathcal{F}^{h H-}\right) \cdot \boldsymbol{n}-\frac{1}{2} S^{+} \Delta(\boldsymbol{u})
$$

with

$$
S^{+}=\max \left(\left|\boldsymbol{v}^{+}\right|+c^{+},\left|\boldsymbol{v}^{-}\right|+c^{-}\right)
$$

In equation (18) $c$ is the sound velocity and $|\boldsymbol{v}|$ is the modulus of the velocity vector at integration point and $\Delta(\boldsymbol{u})=\left(\boldsymbol{u}^{+}-\boldsymbol{u}^{-}\right)$. 


\subsubsection{Li and Gu's fix for the Rusanov flux}

$\mathrm{Li}$ and $\mathrm{Gu}[29]$ described the mechanism underlying several low-Mach fixes for the Roe scheme and based on that description, they developed a low-Mach fix for HLL schemes. Here, we have applied the fix proposed in [29] to the Rusanov flux. It is simply obtained by multiplying the momentum difference term in the momentum equations by the function $f\left(M_{l}\right)$.

\subsubsection{MLS-based shock sensor}

In this work, the Van Albada [47], the Barth and Jespersen [48] and the Venkatakrishnan [49] slope limiters are employed to enforce the Totally Variation Diminishing (TVD) condition of the numerical method for the computation of transonic flows. In order to guarantee that the limiting procedure is not activated for smooth flow regions, thus avoiding the loss of the higher order accuracy of the numerical scheme, we propose to use a selective limiting procedure, based on the multiresolution properties of the Moving Least Squares approximations [50].

Such procedure allows the separation of the high scale components of the solution in order to develop a MLS-based wavelet function of the density that acts as the reference variable. Following [50], the slope limiter algorithm is activated when the following condition is verified

$$
\left|\sum_{j=1}^{n_{I}} \rho_{j}\left(N_{j}^{s_{x}^{H}}(\boldsymbol{x})-N_{j}^{s_{x}^{L}}(\boldsymbol{x})\right)\right|>T_{v}
$$

Setting $s_{x}^{H}=2 s_{x}^{L}$ the term $\sum_{j=1}^{n_{I}} \rho_{j}\left(N_{j}^{s_{x}^{H}}(\boldsymbol{x})-N_{j}^{s_{x}^{L}}(\boldsymbol{x})\right)$ represents the highscale part of the density solution. The high-scale part has a greater value in 
the vicinity of shock waves. The threshold value $T_{v}$ is a problem-dependent parameter, defined as

$$
T_{v}=C_{l c}|\nabla \rho|_{I}\left(A_{I}\right)^{\frac{1}{d}} / \max \left(M_{l}\right)
$$

where $A_{I}$ is the size (area in 2D) of the control volume $I, d$ is the number of dimensions of the problem, $C_{l c}$ is a case-dependent parameter and $\max \left(M_{l}\right)$ is the maximum local Mach number in the computational domain. The range of the $C_{l c}$ parameter is in the interval $[0,0.5]$. If the parameter is chosen as $C_{l c}=0$ the slope-limiter algorithm will be activated in the whole domain of computation. As investigated in [50], a good compromise between robustness and accuracy is obtained for $C_{l c}=0.32$.

\section{Obtaining physical solution using higher-order MLS reconstruc- tions}

In this section we focus on the accuracy properties of the high-order reconstructions by performing a grid refinement study using a sequence of four refined O-type meshes with regular quad cells. Euler equations are used to compute the potential flow past a circular cylinder at Mach numbers ranging from $M_{\infty}=10^{-6}$ to $M_{\infty}=10^{-1}$. High-order MLS reconstructions are achieved up to $4^{\text {th }}$ order.

The coarsest grid, shown in Figure 2, is built from 32 points equally distributed in the circumferential direction and 16 points in the radial direction. Three additional grids $(48 \times 24,64 \times 32$ and $96 \times 48)$ are obtained by refining the coarsest mesh in both directions. The far-field is situated at 40 diameters away from the cylinder. 

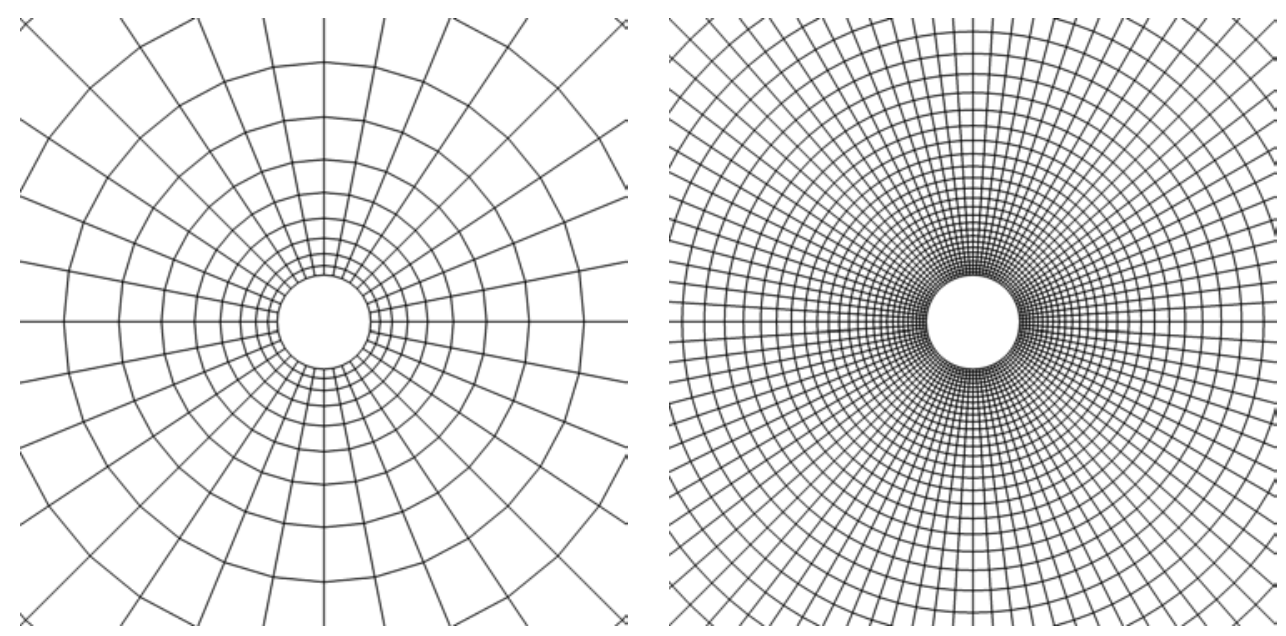

Figure 2: Close view of the coarsest and the finest structured O-grids employed for the computation of the inviscid flow past a circular cylinder test case. The coarsest mesh (left) has $32 \times 16$ elements and the finest (right) $96 \times 48$ elements.

All computations are initialized using a uniform flow, and they are converged until the $L_{2}$ norm of the residuals falls below $10^{-10}$.

\subsection{Roe scheme}

\subsubsection{Numerical experiment}

Here, we investigate the effect of using higher-order MLS reconstruction schemes on the accuracy of low-Mach flows computed using the numerical flux of Roe (named hereafter the ROE-FV-MLS scheme). Freestream mach number is $M_{\infty}=10^{-3}$ and the $96 \times 48$ mesh is employed. Figure 3 presents a comparison of the pressure contours between the $1^{\text {st }}$ order FV scheme and those obtained with the $4^{\text {th }}$ order FV-MLS method.

The low order solution exhibits the known "creep" unphysical solution [46]. For this grid, this problem is circumvented using the high-order scheme. 

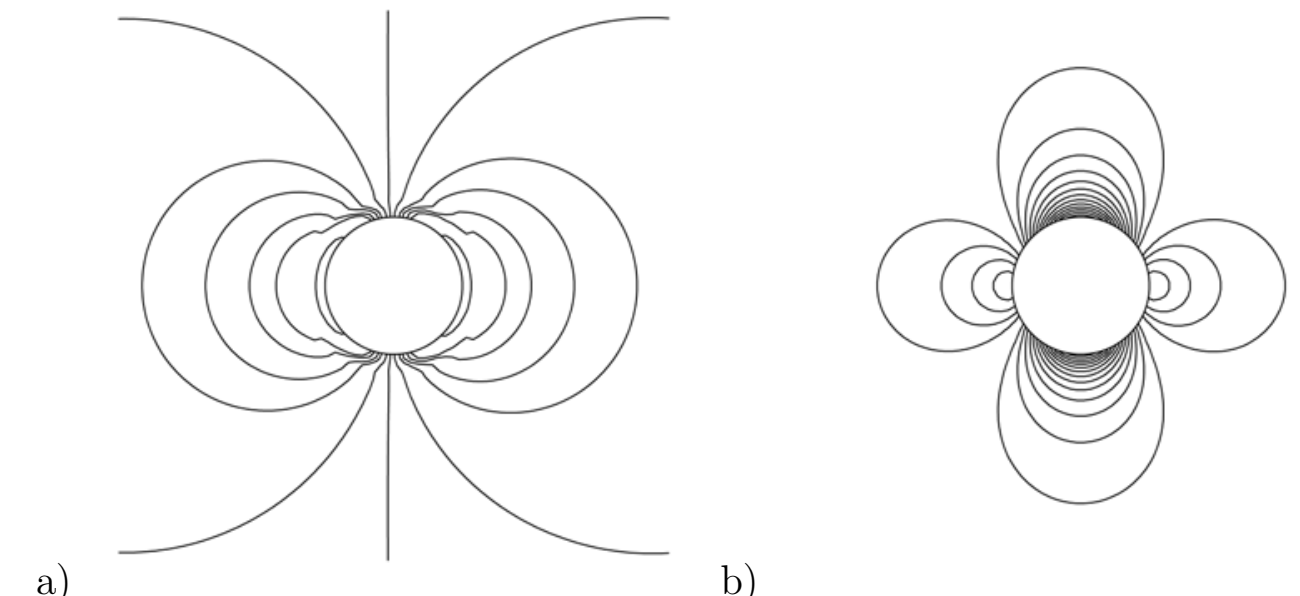

Figure 3: Pressure contours for inviscid flow past a cylinder test case for $M_{\infty}=10^{-3}$. The solution is obtained in the $96 \times 48$ grids by using a first order Roe scheme (a) and by using the $4^{\text {th }}$ order ROE-FV-MLS scheme (b).

Next, we study the order of accuracy of the $4^{\text {th }}$-order ROE-FV-MLS scheme by computing the error in the drag coefficient for $M_{\infty}$ ranging from $10^{-1}$ to $10^{-3}$. Table 1 shows that the formal order of accuracy is recovered for both $M_{\infty}=10^{-1}$ and $M_{\infty}=10^{-2}$. We notice that obtaining physical solution for $M_{\infty}=10^{-3}$ requires the use of a finer grid resolution than for lower Mach numbers. This shows that the increase in the order of a finite volume scheme helps to alleviate the accuracy problem for low-Mach flows. However this procedure is not fully satisfactory since lack of robustness is observed due to grid-dependent results. Note that the same remark holds for Discontinous Galerkin schemes [35].

To get further insight in the behavior of the ROE-FV-MLS scheme at very low Mach numbers, we now compute the normalized pressure fluctuations as 


\begin{tabular}{|c|c|c|c|}
\hline \multicolumn{3}{|c|}{ Fourth order ROE-FV-MLS method } \\
\hline \hline \multirow{4}{*}{ Mach } & Mesh & $C_{D R A G}$ & Order \\
\hline \hline \multirow{7}{*}{$10^{-1}$} & $32 \times 16$ & $4.05 \times 10^{-2}$ & - \\
\cline { 2 - 4 } & $48 \times 24$ & $8.30 \times 10^{-3}$ & 3.91 \\
\cline { 2 - 4 } & $64 \times 32$ & $2.52 \times 10^{-3}$ & 4.15 \\
\cline { 2 - 4 } & $96 \times 48$ & $4.58 \times 10^{-4}$ & 4.20 \\
\hline \multirow{4}{*}{$10^{-2}$} & $32 \times 16$ & $3.93 \times 10^{-1}$ & - \\
\cline { 2 - 4 } & $48 \times 24$ & $7.04 \times 10^{-2}$ & 4.24 \\
\cline { 2 - 4 } & $64 \times 32$ & $1.96 \times 10^{-2}$ & 4.44 \\
\cline { 2 - 4 } & $96 \times 48$ & $3.31 \times 10^{-3}$ & 4.39 \\
\hline \multirow{4}{*}{$10^{-3}$} & $48 \times 24$ & $\left(9.51 \times 10^{-1}\right)$ & - \\
\hline & $64 \times 32$ & $\left(2.48 \times 10^{-1}\right)$ & - \\
\hline & $96 \times 48$ & $3.11 \times 10^{-2}$ & - \\
\hline
\end{tabular}

Table 1: Inviscid flow past a cylinder test case. Accuracy orders for the $4^{\text {th }}$ ROE-FV-MLS scheme for different Mach numbers, where () denotes that the obtained solution is not physical. For $M_{\infty}=10^{-3}$ we have only obtained a physical solution for the finest grid.

$$
p_{\text {norm }}=\frac{p_{\max }-p_{\min }}{p_{\max }}
$$

where $p_{\max }$ and $p_{\min }$ are the maximum and minimum pressures on the com- 
putational domain.

In figure 4 (a), we observe that both $3^{\text {rd }}$ and $4^{\text {th }}$ ROE-FV-MLS numerical simulations, performed on the $32 \times 16$ grid, exhibit pressure fluctuations that are $\mathcal{O}\left(M_{\infty}^{2}\right)$ until a given Mach number.

The comparison in Figure 4 (b) of plots of the pressure fluctuations against $M_{\infty}$ for two grid levels (namely the $32 \times 16$ and the $48 \times 24$ grids) clearly shows the grid dependence of the correct $\mathcal{O}\left(M_{\infty}^{2}\right)$ pressure scaling for a given Mach number.

a)

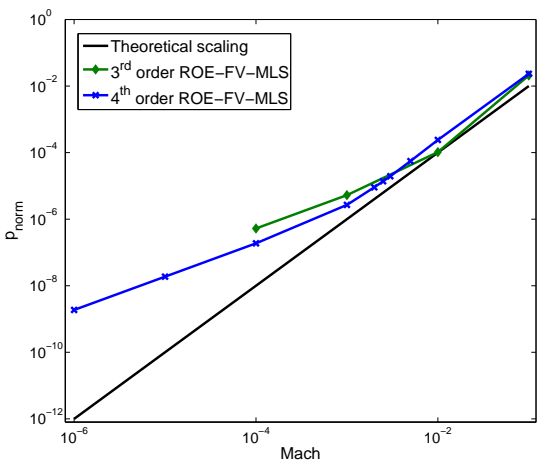

b)

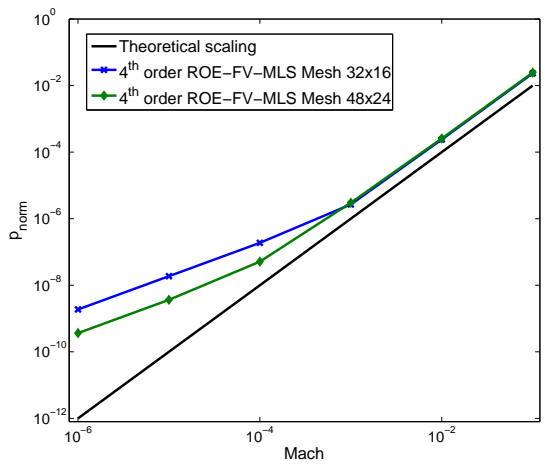

Figure 4: Inviscid flow past a cylinder test case. a) Pressure-Mach scaling for the $3^{\text {rd }}$ and $4^{\text {th }}$ order ROE-FV-MLS scheme in the $32 \times 16$ grid. b) Influence of the grid on the accuracy problem. Pressure-Mach scaling for the $4^{\text {th }}$ order ROE-FV-MLS scheme using different grids.

In [51] it is shown that, for the inviscid low-Mach flow past a cylinder, the first order Roe scheme verifies a scaling with the Mach number of the form $N \sim M_{\infty}^{-1}$, where $N$ is the number of points on the cylinder wall required to obtain a physical solution. We have performed a study of that scaling for the $4^{\text {th }}$ order FV-MLS scheme, and we have obtained a scaling of the form $N \sim M_{\infty}^{-0.388}$. It is clear that increasing the order of the scheme decreases the 
Mach number of the flow that is possible to accurately compute on a given grid, but the Mach dependency is not completely eliminated. Note that the scaling study has been performed considering that a solution is accepted if the relative error in the $\mathcal{O}\left(M_{\infty}^{2}\right)$ scaling of the pressure fluctuations is smaller than a $10 \%$.

\subsubsection{Discussion}

Following $[27,29]$ we can write the dissipation term of the Roe scheme in the $i$-direction as

$$
\boldsymbol{F}_{\boldsymbol{d}}=-\frac{1}{2}\left\{|U|\left[\begin{array}{c}
\Delta \rho \\
\Delta(\rho u) \\
\Delta(\rho v) \\
\Delta(\rho E)
\end{array}\right]+\delta U\left[\begin{array}{c}
\rho \\
\rho u \\
\rho v \\
H
\end{array}\right]+\delta p\left[\begin{array}{c}
0 \\
n_{x} \\
n_{y} \\
U
\end{array}\right]\right\}
$$

where $U=n_{x} u+n_{y} v$ is the normal component of the velocity. Contributions to the modification to the interface fluxes (terms of $\delta U$ ) and to the modification to the interface pressure (terms of $\delta p$ ) can be written as

$$
\begin{aligned}
& \delta U=(c-|U|) \frac{\Delta p}{\rho c^{2}}+\frac{U}{c} \Delta U \\
& \delta p=(c-|U|) \rho \Delta U+\frac{U}{c} \Delta p
\end{aligned}
$$

In $[14,27,29]$ it is shown that the term $c \rho \Delta U$ of equation (24) associated to the dissipation of the momentum equation is the responsible of the accuracy problem. Rieper's fix (and others such as those of [23, 28]) modify this term by replacing the velocity of sound $c$ by a modified velocity $c^{\prime}=f(M) c$, that reduces the product $c \rho \Delta U$, and obtains the right asymptotic behavior of the 
discrete scheme. In particular, Rieper's fix modifies the dissipation term as follows

$$
\begin{aligned}
& \delta U=(c-|U|) \frac{\Delta p}{\rho c^{2}}+\frac{U}{c} f(M) \Delta U \\
& \delta p=\left(c^{\prime}-f(M)|U|\right) \rho \Delta U+\frac{U}{c} \Delta p
\end{aligned}
$$

When the order of the numerical scheme is increased (or the grid is refined), jumps of the variable at the interfaces are reduced. Thus, the product $c \rho \Delta U$ is also reduced and the accuracy problem is alleviated. Following [29], the key to correct the accuracy problem is to set $c^{\prime} \leq \mathcal{O}\left(c^{0}\right)$. Increasing the order only, the reduction of $\Delta U$ for a given order in a given grid may not be enough to eliminate completely the accuracy problem, since the first-order artificial-viscosity term $c \rho \Delta U$ is still there in the asymptotic limit.

\subsubsection{ROE-FV-MLS results with Rieper's fix}

As a consequence of the previous analysis, we now investigate the combination of the high-order ROE-FV-MLS scheme with the Rieper's fix presented in section 2.2.2. Figure 5 shows the contours of entropy production for the $M=10^{-3}$ inviscid flow past a cylinder test case. In this example, all the entropy generated is due to the numerical discretization. It is observed that the entropy is transported by convection for all the schemes. Moreover the entropy generation is considerably reduced when using the low Mach fix. As expected, increasing the order further reduce the entropy generation.

Next, we perform an accuracy analysis by computing the error in the drag coefficient for $M_{\infty}=10^{-1}, M_{\infty}=10^{-2}$ and $M_{\infty}=10^{-3}$. It can be clearly seen in Table 2 that the convergence orders are successfully recovered for 


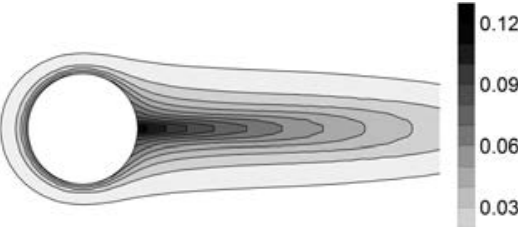

a)

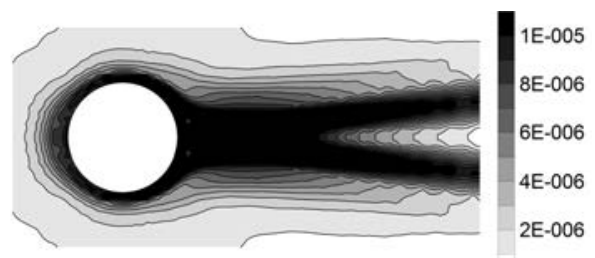

c)

0

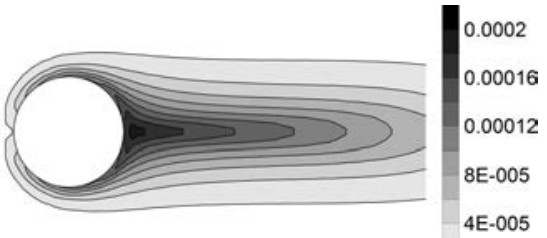

b)
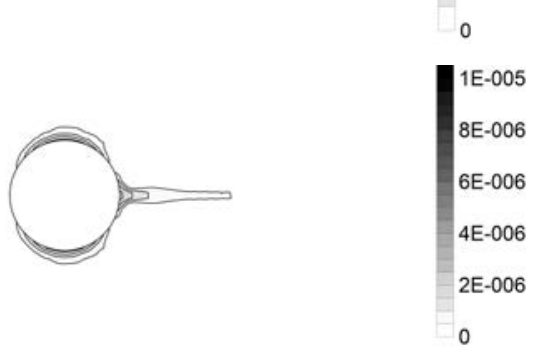

Figure 5: Contours of entropy production and transport for the $M=10^{-3}$ inviscid flow past a cylinder test case $96 \times 48$ grid. a) First order-Roe scheme. b) First-order Roe scheme with Rieper's fix. c) $4^{\text {th }}$ order ROE-FV-MLS scheme with no fix. d) $4^{\text {th }}$ order ROE-FV-MLS scheme with Rieper's fix.

all the Mach numbers considered. Contrary to the high-order scheme with no fix case, the use of the Rieper's fix with the high-order scheme allows physical solutions whatever the grid size (as the right asymptotic behaviour is recovered), thus removing the Mach dependency problem.

As for the no fix case, we analyze the pressure scaling for low-Mach computations with the high-order scheme with the low Mach fix of Rieper. Figure 6 shows that when the low-Mach fix is used, the correct $\mathcal{O}\left(M^{2}\right)$ scaling of the pressure fluctuations is recovered for all the computations.

As an illustration purpose, the pressure field obtained using a $4^{\text {th }}$ order ROE-FV-MLS scheme with Rieper's Fix at $M_{\infty}=10^{-6}$ for the mesh $32 \times 16$ is plotted in Figure 7. We notice that the numerical solution, which is free from 


\begin{tabular}{|c|c|c|c|c|c|}
\hline & & \multicolumn{2}{|c|}{ Third order } & \multicolumn{2}{|c|}{ Fourth order } \\
\hline Mach & Mesh & $C_{D R A G}$ & Order & $C_{D R A G}$ & Order \\
\hline \multirow{4}{*}{$10^{-1}$} & $32 \times 16$ & $1.56 \times 10^{-2}$ & - & $1.96 \times 10^{-2}$ & - \\
\hline & $48 \times 24$ & $2.61 \times 10^{-3}$ & 4.41 & $2.92 \times 10^{-3}$ & 4.70 \\
\hline & $64 \times 32$ & $1.05 \times 10^{-3}$ & 3.17 & $8.49 \times 10^{-4}$ & 4.30 \\
\hline & $96 \times 48$ & $2.68 \times 10^{-4}$ & 3.37 & $1.69 \times 10^{-4}$ & 3.98 \\
\hline \multirow{4}{*}{$10^{-2}$} & $32 \times 16$ & $7.88 \times 10^{-3}$ & - & $1.21 \times 10^{-2}$ & - \\
\hline & $48 \times 24$ & $1.18 \times 10^{-3}$ & 4.69 & $1.63 \times 10^{-3}$ & 4.95 \\
\hline & $64 \times 32$ & $5.61 \times 10^{-4}$ & 2.58 & $4.46 \times 10^{-4}$ & 4.50 \\
\hline & $96 \times 48$ & $1.59 \times 10^{-4}$ & 3.11 & $8.84 \times 10^{-5}$ & 4.00 \\
\hline \multirow{4}{*}{$10^{-3}$} & $32 \times 16$ & $6.48 \times 10^{-3}$ & - & $1.08 \times 10^{-2}$ & - \\
\hline & $48 \times 24$ & $8.76 \times 10^{-4}$ & 4.94 & $1.36 \times 10^{-3}$ & 5.10 \\
\hline & $64 \times 32$ & $4.50 \times 10^{-4}$ & 2.31 & $3.58 \times 10^{-4}$ & 4.65 \\
\hline & $96 \times 48$ & $1.34 \times 10^{-4}$ & 2.99 & $7.05 \times 10^{-5}$ & 4.01 \\
\hline
\end{tabular}

Table 2: Inviscid flow past a cylinder test case. Accuracy orders for the $3^{\text {rd }}$ and $4^{\text {th }}$ order ROE-FV-MLS scheme with Rieper's Fix for different Mach numbers.

artificial wake downstream of the cylinder, presents a perfectly symmetric flow with respect to the coordinates axis. 


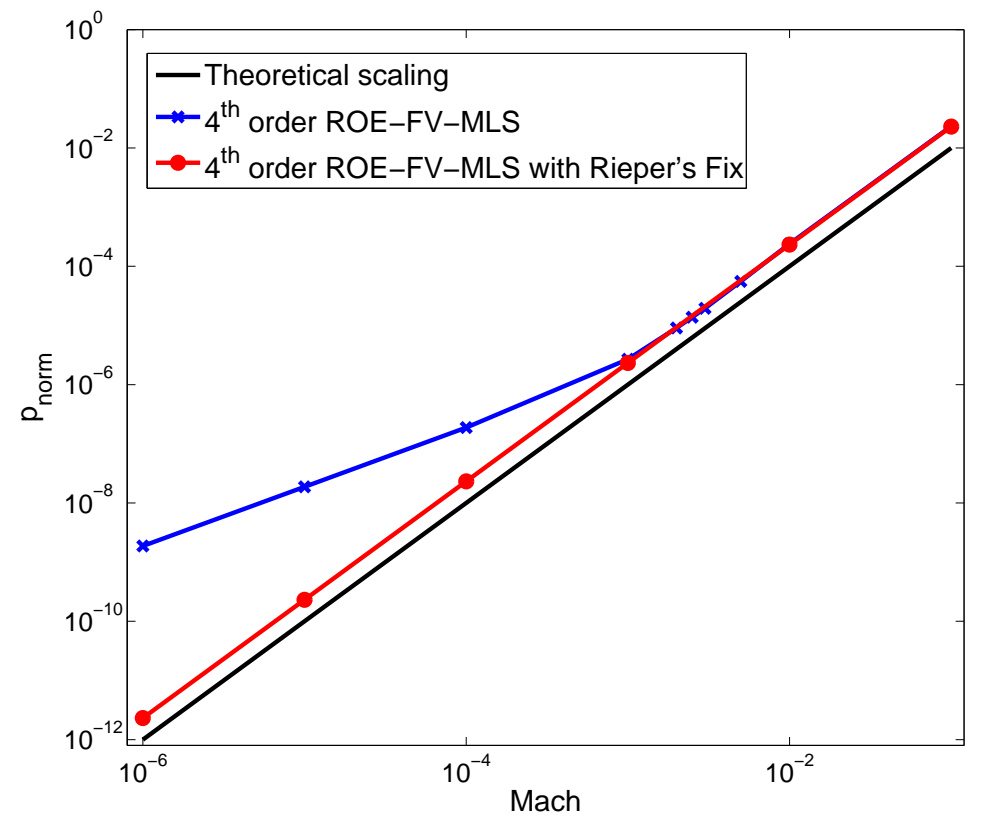

Figure 6: Inviscid flow past a cylinder test case. Pressure-Mach scaling for the $4^{\text {th }}$ order ROE-FV-MLS scheme with Rieper's Fix in the $32 \times 16$ grid. 


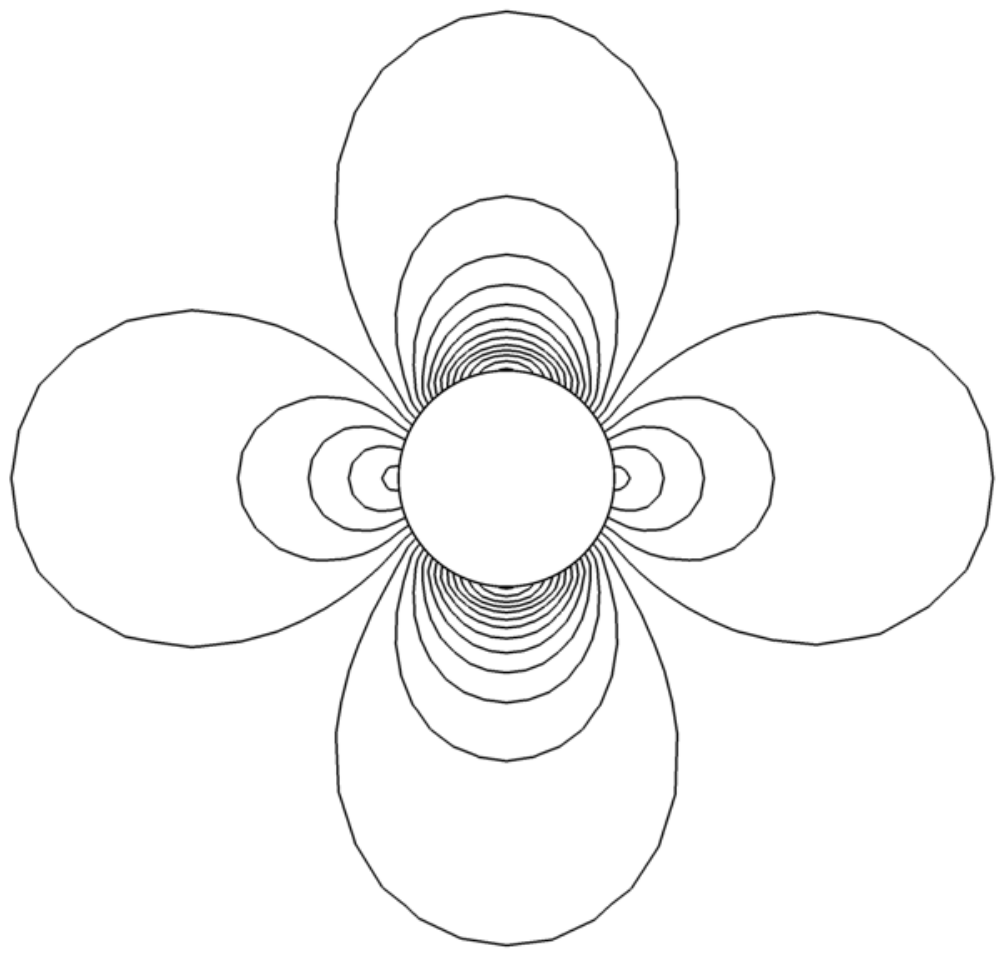

Figure 7: Inviscid flow past a cylinder test case. Pressure contours for $M_{\infty}=10^{-6}$. The solution is obtained in the $32 \times 16$ grid by using the $4^{\text {th }}$ order ROE-FV-MLS scheme and the low-Mach fix of Rieper. 


\subsubsection{Fix with modification of the tangential velocity}

The low Mach fix developed by Rieper [25] only modifies the normal velocity component. It is interesting to investigate if an increase in accuracy in low Mach conditions could be obtained if we modify both, the normal and the tangential velocity component. To this end, the term $\tilde{\alpha}_{2}$ in (13) is written as

$$
\tilde{\alpha}_{2}=\frac{\tilde{\rho} f\left(M_{l}\right)}{\tilde{c}}\left[\Delta(v) n_{x}-\Delta(u) n_{y}\right]
$$

It is observed in figure 8 that entropy production decreases when the low Mach fix is applied to the normal and tangential velocity components compared to solution computed without fix (Figure 5).

a)

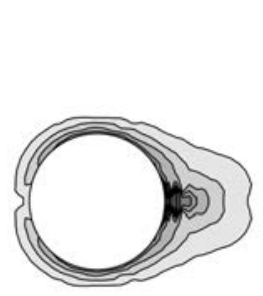

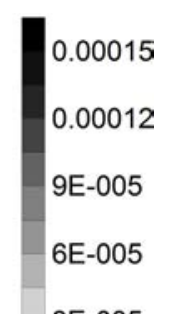

3E-005

b)

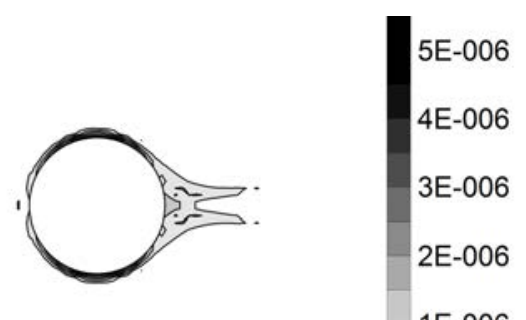

$1 \mathrm{E}-006$

0

Figure 8: Contours of entropy production and transport for the $M=10^{-3}$ inviscid flow past a cylinder test case $96 \times 48$ grid with low Mach fix correcting both the normal and tangential velocities using a) First-order Roe scheme , b) $4^{\text {th }}$ ROE-FV-MLS scheme.

Table 3 shows that the formal order of accuracy of the high order scheme is not affected by this modification. In most cases, the drag coefficient is reduced when the fix is applied to the tangential velocity component compared to the Rieper's fix. 


\begin{tabular}{|c|c|c|c|c|c|}
\hline & & \multicolumn{2}{|c|}{ Third order } & \multicolumn{2}{|c|}{ Fourth order } \\
\hline Mach & Mesh & $C_{D R A G}$ & Order & $C_{D R A G}$ & Order \\
\hline \multirow{4}{*}{$10^{-1}$} & $32 \times 16$ & $1.46 \times 10^{-2}$ & - & $1.62 \times 10^{-2}$ & - \\
\hline & $48 \times 24$ & $2.63 \times 10^{-3}$ & 4.23 & $2.59 \times 10^{-3}$ & 4.53 \\
\hline & $64 \times 32$ & $9.94 \times 10^{-4}$ & 3.38 & $7.74 \times 10^{-4}$ & 4.19 \\
\hline & $96 \times 48$ & $2.36 \times 10^{-4}$ & 3.54 & $1.52 \times 10^{-4}$ & 4.01 \\
\hline \multirow{4}{*}{$10^{-2}$} & $32 \times 16$ & $6.69 \times 10^{-3}$ & - & $8.15 \times 10^{-3}$ & - \\
\hline & $48 \times 24$ & $1.23 \times 10^{-3}$ & 4.17 & $1.27 \times 10^{-3}$ & 4.59 \\
\hline & $64 \times 32$ & $5.00 \times 10^{-4}$ & 3.14 & $3.65 \times 10^{-4}$ & 4.33 \\
\hline & $96 \times 48$ & $1.16 \times 10^{-4}$ & 3.61 & $6.56 \times 10^{-5}$ & 4.23 \\
\hline \multirow{4}{*}{$10^{-3}$} & $32 \times 16$ & $5.36 \times 10^{-3}$ & - & $6.83 \times 10^{-3}$ & - \\
\hline & $48 \times 24$ & $9.69 \times 10^{-4}$ & 4.22 & $1.03 \times 10^{-3}$ & 4.67 \\
\hline & $64 \times 32$ & $4.07 \times 10^{-4}$ & 3.02 & $2.85 \times 10^{-4}$ & 4.46 \\
\hline & $96 \times 48$ & $9.44 \times 10^{-5}$ & 3.60 & $5.35 \times 10^{-5}$ & 4.13 \\
\hline
\end{tabular}

Table 3: Inviscid flow past a cylinder test case. Accuracy orders for the $3^{\text {rd }}$ and $4^{\text {th }}$ order ROE-FV-MLS scheme correcting the normal and tangential velocities for different Mach numbers.

\subsection{Rusanov scheme}

In this section, we extend the previous study to the FV-MLS solver with Rusanov flux (namely the RUS-FV-MLS method). As shown in Table 4, we note that the $4^{\text {th }}$ order reconstruction scheme gives the expected order of 
convergence for Mach numbers $M_{\infty}=10^{-1}$ and $M_{\infty}=10^{-2}$. Note however that we were unable to obtain a physical solution for Mach numbers below $10^{-2}$.

Next, we analyze the results of the RUS-FV-MLS scheme combined with the Li and Gu's low-Mach fix presented in section 2.2.4. Figure 9 shows the pressure contours obtained for $M_{\infty}=10^{-2}$ on a $96 \times 48$ grid. Although the $4^{\text {th }}$ high-order scheme does not give a fully symmetrical solution, it can be observed that the solution is greatly improved compared to its low order counterpart. It is important to note that, for $1^{\text {st }}$ order HLL schemes, the low-Mach fix does not solve completely the checkerboard problem [29]. On the contrary, the solution of the $4^{\text {th }}$ order RUS-FV-MLS scheme with Li and Gu's fix is free from checkerboard.

In order to explain this, we consider the dissipation term of the Rusanov scheme [29]

$$
\boldsymbol{F}_{d}=-\frac{1}{2} S^{+}\left[\begin{array}{c}
\Delta \rho \\
\Delta(\rho u) \\
\Delta(\rho v) \\
\Delta(\rho E)
\end{array}\right]
$$

with

$$
S^{+}=\max \left(\left|\boldsymbol{v}^{+}\right|+c^{+},\left|\boldsymbol{v}^{-}\right|+c^{-}\right)
$$

The checkerboard problem is due to the density difference term $\Delta \rho$ in (28) which is related to the pressure difference term by $\Delta \rho=\frac{\Delta p}{c^{2}}$. Li and Gu's fix consists in modifying the dissipation term as 


\begin{tabular}{|c|c|c|c|}
\hline \multicolumn{4}{|c|}{ Fourth order RUS-FV-MLS method } \\
\hline Mach & Mesh & $C_{D R A G}$ & Order \\
\hline \multirow{4}{*}{$10^{-1}$} & $32 \times 16$ & $2.36 \times 10^{-1}$ & - \\
\hline & $48 \times 24$ & $4.85 \times 10^{-2}$ & 3.90 \\
\hline & $64 \times 32$ & $1.41 \times 10^{-2}$ & 4.30 \\
\hline & $96 \times 48$ & $2.09 \times 10^{-3}$ & 4.71 \\
\hline \multirow{4}{*}{$10^{-2}$} & $32 \times 16$ & $1.04 \times 10^{0}$ & - \\
\hline & $48 \times 24$ & $3.49 \times 10^{-1}$ & 2.70 \\
\hline & $64 \times 32$ & $1.24 \times 10^{-1}$ & 3.59 \\
\hline & $96 \times 48$ & $2.14 \times 10^{-2}$ & 4.34 \\
\hline \multirow{4}{*}{$10^{-3}$} & $32 \times 16$ & $\left(4.51 \times 10^{0}\right)$ & - \\
\hline & $48 \times 24$ & $\left(1.56 \times 10^{0}\right)$ & - \\
\hline & $64 \times 32$ & $\left(7.20 \times 10^{-1}\right)$ & - \\
\hline & $96 \times 48$ & $\left(1.82 \times 10^{-1}\right)$ & - \\
\hline
\end{tabular}

Table 4: Inviscid flow past a cylinder test case. Accuracy orders for the $4^{\text {th }}$ order RUSFV-MLS scheme for different Mach numbers. Quantities in () denotes that the obtained solution is non-physical

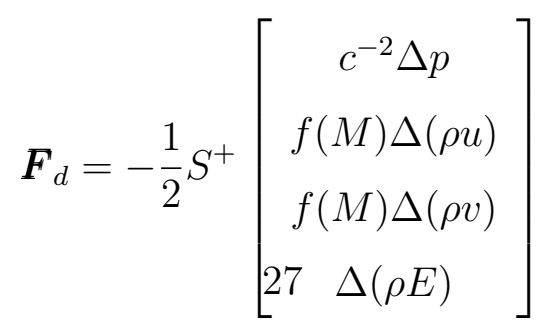


where $S^{+}=\mathcal{O}(c)$ and $S^{+} \Delta p c^{-2}=\mathcal{O}\left(c^{-1}\right)$. In [29] it is shown that when product of the terms multiplying $\Delta p$ in the numerical dissipation term of the continuity equation is of the order $\mathcal{O}\left(c^{-1}\right)$ a weak checkerboard is allowed. Increasing the order of the numerical scheme does not modify the $\mathcal{O}\left(c^{-1}\right)$ character of this product, but as $\Delta p$ is reduced, the importance of the product $S^{+} \Delta p c^{-2}$ is also reduced.

a)

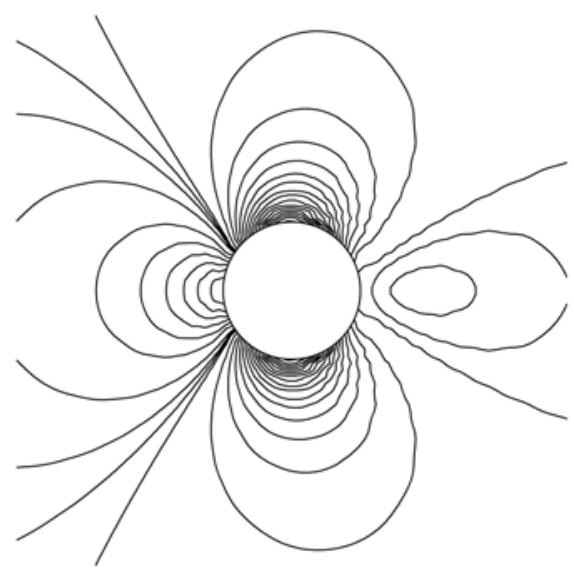

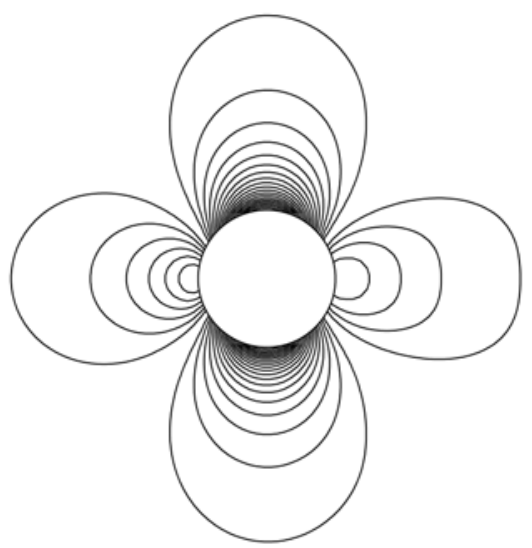

b)

Figure 9: Inviscid flow past a cylinder test case. Pressure contours for $M_{\infty}=10^{-2}$. The solution is obtained in the $96 \times 48$ grid by using the Li and Gu's low-Mach fix with a first order FV Rusanov scheme (a) and the $4^{\text {th }}$ order RUS-FV-MLS scheme. It is observed that the first order scheme presents a weak checkerboard that is removed with the use of the high-order scheme.

Table 5 shows that the use of the Li and Gu's fix for the Rusanov scheme allows to recover the expected order of accuracy for all the tested Mach numbers. Recall that, with the grids considered, it was not possible to obtain a physical solution for a Mach number below $M_{\infty}=10^{-2}$ without using the fix (Table 4). 


\begin{tabular}{|c|c|c|c|}
\hline \multicolumn{4}{|c|}{ Fourth order Rusanov scheme with Li and Gu's Fix } \\
\hline Mach & Mesh & $C_{D R A G}$ & Order \\
\hline \multirow{4}{*}{$10^{-1}$} & $32 \times 16$ & $6.53 \times 10^{-2}$ & - \\
\hline & $48 \times 24$ & $1.04 \times 10^{-2}$ & 4.54 \\
\hline & $64 \times 32$ & $2.84 \times 10^{-3}$ & 4.51 \\
\hline & $96 \times 48$ & $4.40 \times 10^{-4}$ & 4.59 \\
\hline \multirow{4}{*}{$10^{-2}$} & $32 \times 16$ & $5.31 \times 10^{-2}$ & - \\
\hline & $48 \times 24$ & $8.46 \times 10^{-3}$ & 4.53 \\
\hline & $64 \times 32$ & $2.30 \times 10^{-3}$ & 4.52 \\
\hline & $96 \times 48$ & $3.47 \times 10^{-4}$ & 4.67 \\
\hline \multirow{4}{*}{$10^{-3}$} & $32 \times 16$ & $5.15 \times 10^{-2}$ & - \\
\hline & $48 \times 24$ & $8.14 \times 10^{-3}$ & 4.55 \\
\hline & $64 \times 32$ & $2.20 \times 10^{-3}$ & 4.55 \\
\hline & $96 \times 48$ & $3.41 \times 10^{-4}$ & 4.58 \\
\hline
\end{tabular}

Table 5: Inviscid flow past a cylinder test case. Accuracy orders for the $4^{\text {th }}$ order RUSFV-MLS scheme with Li and Gu's fix for different Mach numbers.

Figure 10 shows that the $4^{\text {th }}$ RUS-FV-MLS scheme loses the right $\mathcal{O}\left(M_{\infty}^{2}\right)$ pressure fluctuations from a greater value of the Mach number than those obtained for the ROE-FV-MLS scheme. As for the Roe case, the low Mach fix changes the terms $S^{+} \Delta(\rho u)$ and $S^{+} \Delta(\rho v)$. These terms play a similar role 
to $c \rho \Delta U$ in the Roe scheme. Using the high-order approach, the increase in the order results in a decrease of the $\Delta \boldsymbol{u}$ term of equation (28), reducing the value of the product. However, when the low-Mach fix is used, the accuracy problem is solving since $S^{+}=\mathcal{O}\left(c^{0}\right)$.

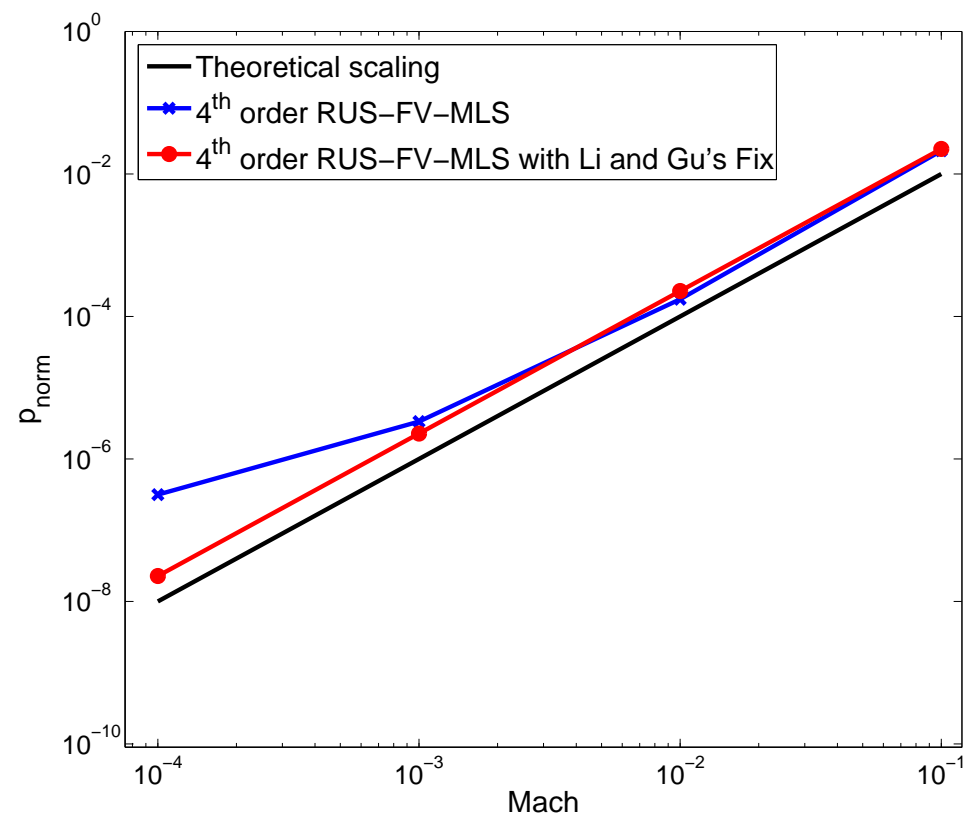

Figure 10: Inviscid flow past a cylinder test case. Comparison of the pressure-Mach scaling for the $4^{\text {th }}$ order RUS-FV-MLS scheme with and without the Li and Gu's Fix for the $32 \times 16$ grid.

In figure 11 we show the distribution of the entropy contours for the $M=10^{-2}$ inviscid flow past a cylinder test case. It is observed that for the first-order Rusanov scheme without low Mach fix, the entropy presents a dissipative transport. When the fix is applied, the entropy is convected. The same behavior is obtained when the fourth-order scheme is applied with and without fix. However, the use of the low Mach fix dramatically reduces the 
entropy production (figures 11 (a) and (b)).

a)
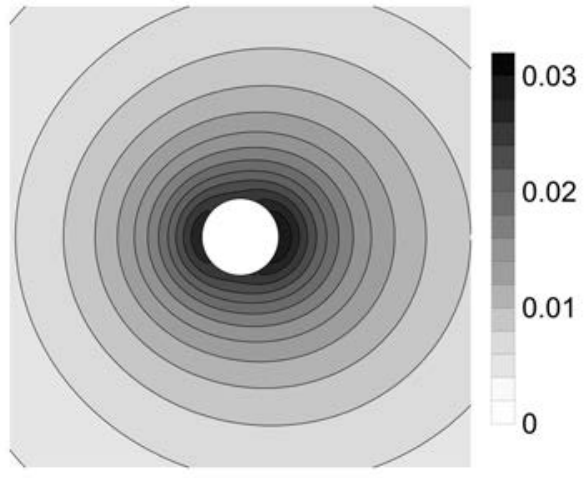

b)

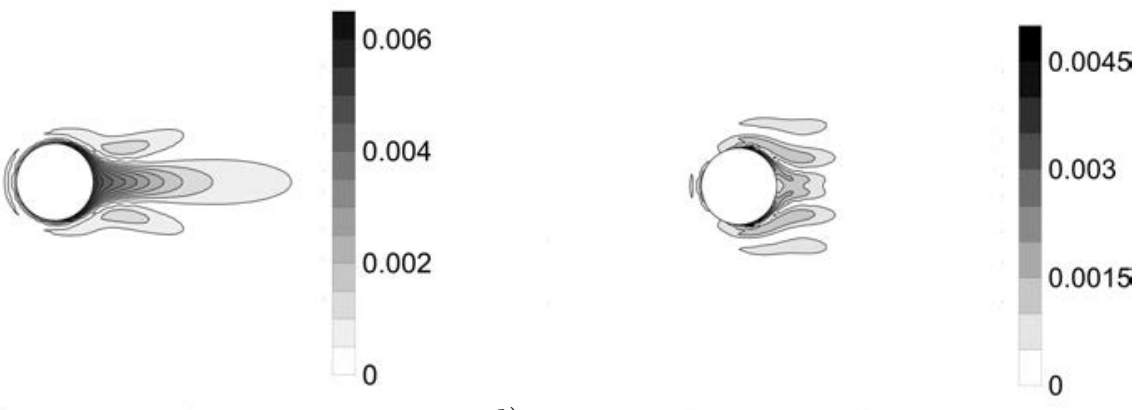

c)

d)

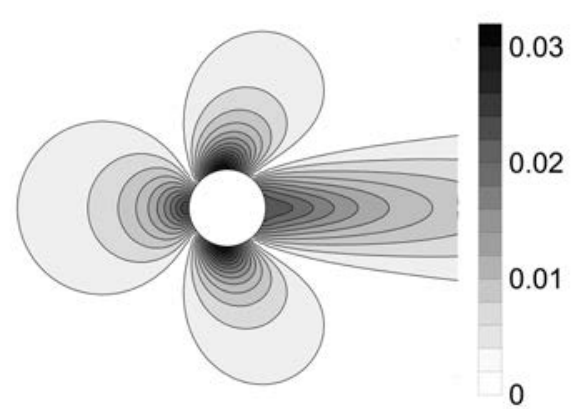

Figure 11: Contours of entropy production and transport for the $M=10^{-2}$ inviscid flow past a cylinder test case $96 \times 48$ grid. a) First order-Rusanov scheme. b) First-order Rusanov scheme with Li and Gu's fix. c) $4^{\text {th }}$ order RUS-FV-MLS scheme with no fix. d) $4^{\text {th }}$ order RUS-FV-MLS scheme with Rieper's fix.

\section{Low-Mach fixes and slope limiters}

In this section, we combine the use of the previously investigated LowMach fixes with slope limiters.

The idea behind the slope limiters, is to create a limited higher-order (piecewise linear discontinuous) reconstruction of the solution with a limited 
gradient that enforces the TVD condition. For example, the limited secondorder reconstruction can be expressed as

$$
\boldsymbol{u}_{I}^{h b}(\boldsymbol{x})=\boldsymbol{u}_{I}^{h}+\chi_{I} \boldsymbol{\nabla} \boldsymbol{u}_{I}^{h} \cdot\left(\boldsymbol{x}-\boldsymbol{x}_{I}\right)
$$

where the value $\chi_{I}$ is obtained with the slope limiter.

Ideally, the limiter should not be active in smooth or low Mach regions, in order to keep the accuracy of the high-order scheme. To illustrate this point, we compute in the following the inviscid flow past a circular cylinder at $M_{\infty}=10^{-3}$.

\subsection{Roe scheme with Rieper's fix}

Here, we consider the Roe scheme with Rieper's fix and a fourth-order MLS reconstruction scheme. The unstructured mesh shown in Figure 12 has 64 elements on the cylinder surface and the total number of elements is 2320 .

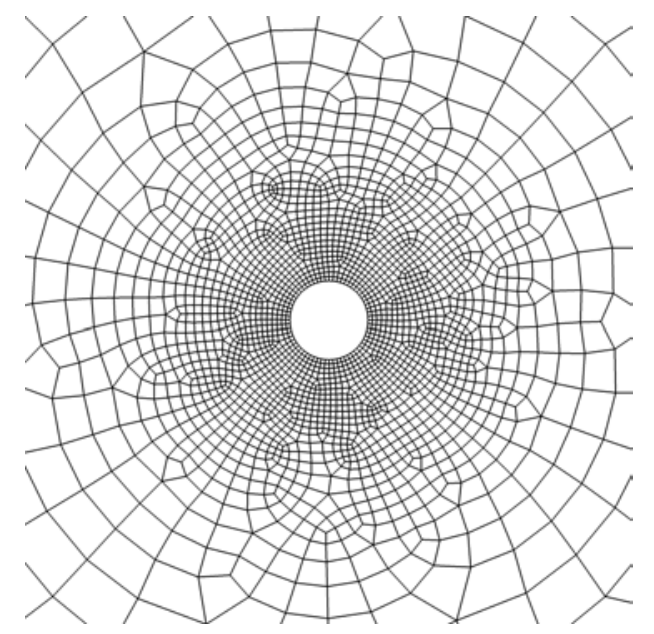

Figure 12: Close view of the unstructured O-grid employed for the computation of the inviscid flow past a circular cylinder test case using slope limiters. 
Figures 13 a) and b) present the pressure contours obtained by combining the Barth and Jespersen [48] and the Venkatakrishnan [49] slope limiters with Rieper's fix. As suggested in [52], Figure 13 a) shows that spurious oscillations are avoided using the Venkatakrishnan limiter.

The accuracy of the fix is clearly affected by the use of these limiting strategies. The undesirable behaviour of the Barth and Jespersen limiter is due to the unnecessary activation of the limiter in smooth regions as clearly observed in figure $13 \mathrm{c}$ ). The good result obtained using the Venkatakrishnan limiter with Rieper's fix is explained by the fact that only few cells close to the cylinder are selected for the application of the slope limiting procedure (figure $13 \mathrm{~d})$ ).

To avoid such problems for any limiter, whatever the considered type of slope limiter, we apply the MLS-based shock sensor strategy presented in section 2.2.5 on the Barth-Jespersen limiter with Rieper's low-Mach. Figure 14 shows that the MLS sensor perfectly succeeds in prevented unwanted activation of the limiter for smooth flow in comparison with figure 13 a).

Table 6 summarizes the drag coefficient $C_{D R A G}$ obtained with the different slope limiting strategies. We observe that Barth-Jespersen and VanAlbada slope limiters give unacceptable values of $C_{D R A G}$ compared to the reference solution without slope limiter. On the contrary, the use of the MLS-based shock sensor allow the recover the reference value for $C_{D R A G}$. Again, the difference between the reference solution and the solution obtained with Venkatakrishnan limiter is due to the unnecessary activation of the slope limiter. 


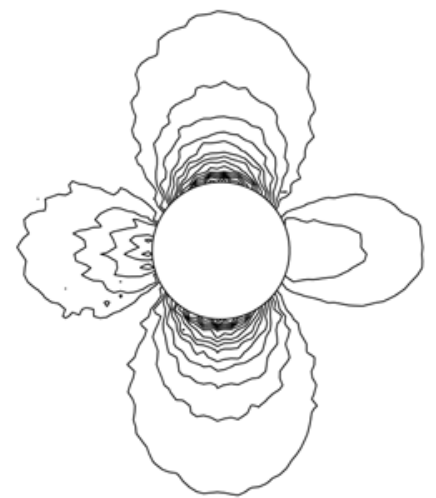

a)

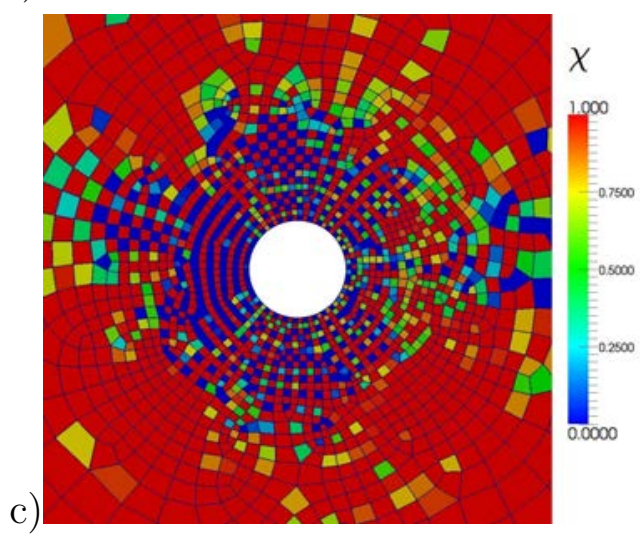

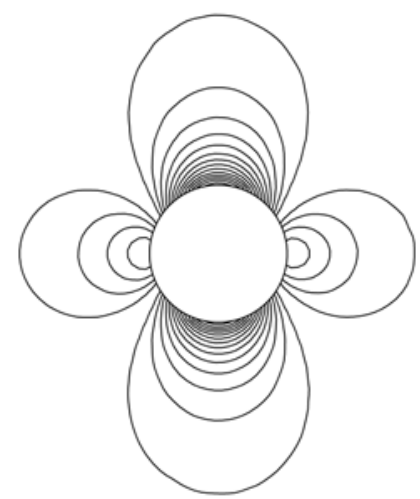

b)

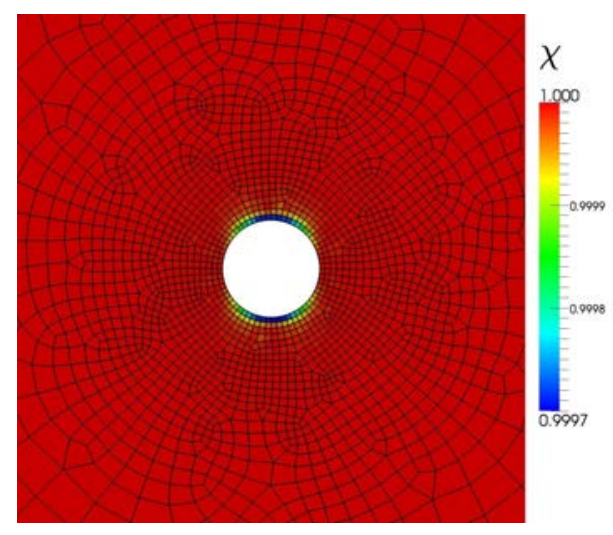

Figure 13: Inviscid $M_{\infty}=10^{-3}$ flow past a cylinder on an unstructured grid. Pressure contours with Rieper's low-Mach fix using Barth-Jespersen limiter (a) and Venkatakrishnan limiter (b). Limited cells using Barth-Jespersen limiter (c) and Venkatakrishnan limiter (d). 


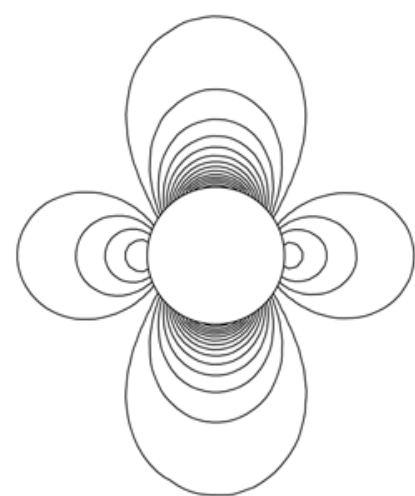

a)

Figure 14: Inviscid $M_{\infty}=10^{-3}$ flow past a cylinder on an unstructured grid. Pressure contours with Barth-Jespersen limiter with Rieper's low-Mach fix and MLS-based sensor (a). Activated cells using Barth-Jespersen limiter and MLS-based sensor with $\left(C_{l c}=0.32\right)$.

\begin{tabular}{lc}
\hline Limiter & $C_{D R A G}$ \\
\hline Barth-Jespersen [48] & $8.64 \times 10^{-2}$ \\
Van Albada [47] & $2.37 \times 10^{-1}$ \\
Venkatakrishnan [49] & $3.43 \times 10^{-3}$ \\
Barth-Jespersen + MLS-based sensor & $3.34 \times 10^{-3}$ \\
Van Albada + MLS-based sensor & $3.34 \times 10^{-3}$ \\
\hline No limiter & $3.34 \times 10^{-3}$ \\
\hline
\end{tabular}

Table 6: Inviscid $M_{\infty}=10^{-3}$ flow past a cylinder on an unstructured grid. Comparison of the drag coefficient for different slope limiters. The solutions are obtained with a fourth order Rieper's Fix Roe FV-MLS scheme on an unstructured mesh. 


\subsection{Rusanov scheme with Li and Gu's fix}

The application of slope limiters to the low-Mach fix of Li and Gu produces the same problems than those reported in the previous section for the Rieper's fix. In Figure 15 a) and b) we observe that the use of BarthJespersen and Van Albada limiters with the low Mach fix gives spurious pressure oscillations. As in the Roe's case, the use of the Venkatakrishnan limiter or the MLS-based sensor does not introduce spurious oscillations, as it is shown in Figure 15 c) and Figure 15 d).

\section{Unsteady transonic viscous flow over a circular cylinder}

This last example aims to highlight the robustness and accuracy capabilities of the present high-order solver for all-speed flows. To this end, we compute the transonic viscous flow past a circular cylinder at free-stream Mach number equal to 0.8. This configuration involves complex viscous-shock interactions and vortex shedding in the vicinity of the wake as investigated experimentally in [53].

The Reynolds number based on a diameter of the cylinder $D=1 \mathrm{~m}$ is $R e=166.000$. For this example no turbulence model has been used, according to the approach adopted in $[54,55]$.

The outer boundary of the O-topology mesh is located at a distance of 200 diameters from the center of the cylinder. The cylinder surface is discretized with 720 control volumes. The normal distance of the first centroid to the cylinder wall is $y_{n}=2.85 \times 10^{-4} D$. The total number of control volumes is 206.150 .

Figure 16 present the magnitude of the temperature gradient computed 


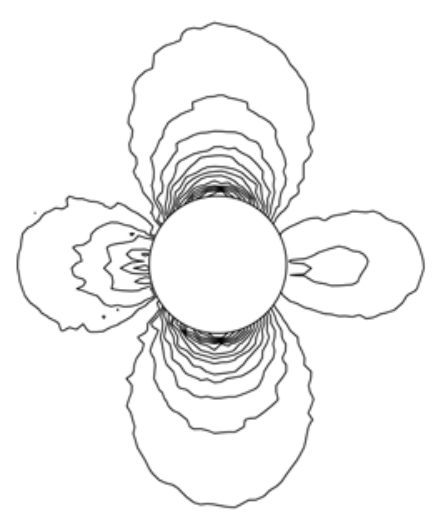

a)

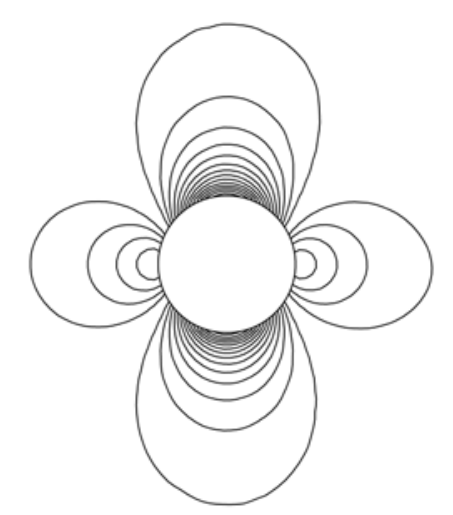

c)

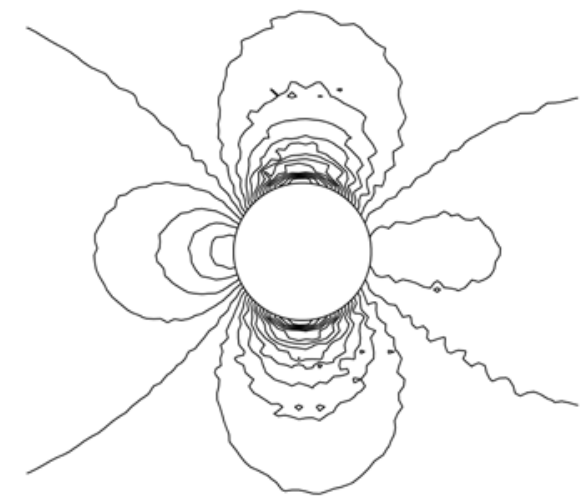

b)

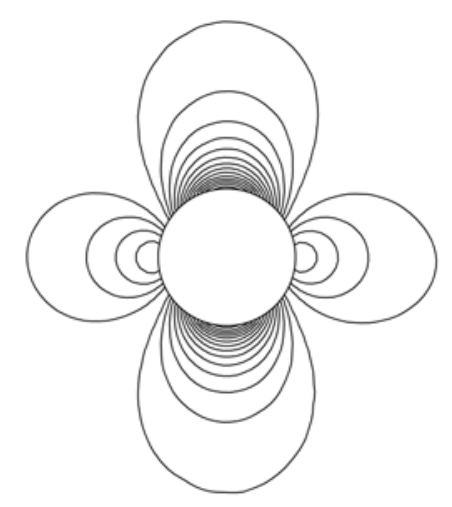

d)

Figure 15: Inviscid $M_{\infty}=10^{-3}$ flow past a cylinder on an unstructured grid. Pressure contours. Rusanov's low-Mach fix with: Barth-Jespersen limiter (a), Van Albada limiter (b). Rieper's low-Mach fix with Barth-Jespersen limiter (c) and with Van Albada limiter and MLS-based sensor (d).

for $t^{*}=t U_{\text {inlet }} / D$ equal to 49.93 . This result was obtained by using a $3^{r d}$ order ROE-FV-MLS scheme with the Rieper's fix. The MLS-based shock sensor is applied to the Van Albada slope limiter. We clearly observe the complex viscous-shock interaction pattern near the cylinder as experimen- 
tally investigated in [53].

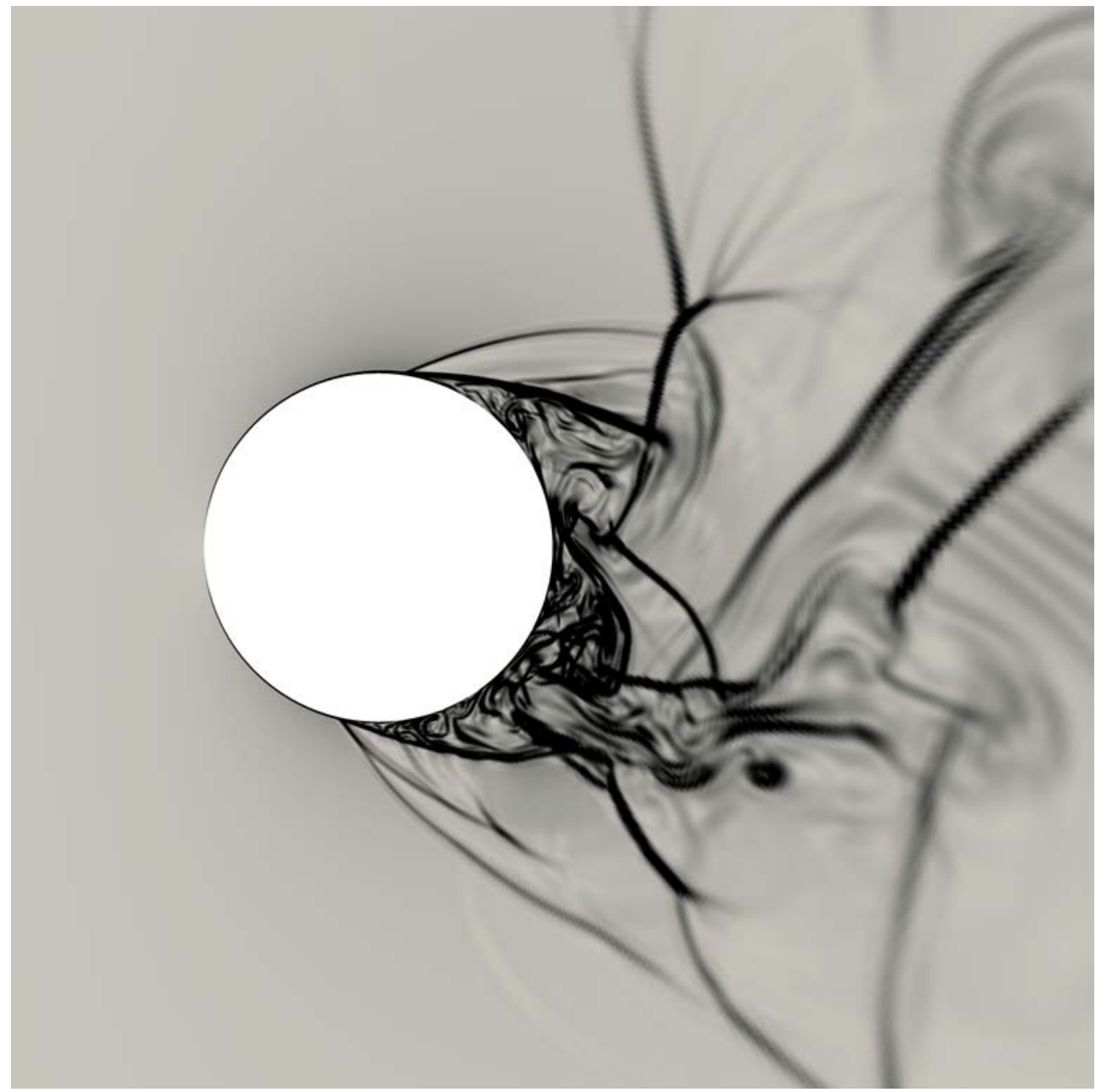

Figure 16: Unsteady transonic viscous flow over a circular cylinder. Magnitude of the temperature gradient near the cylinder for $t^{*}=49.93$.

The computation of the Mach field at the same non-dimensional time $\left(t^{*}=49.93\right)$ in Figure 17 shows that a von Kármán vortex street is formed after the viscous-shock interaction region. 


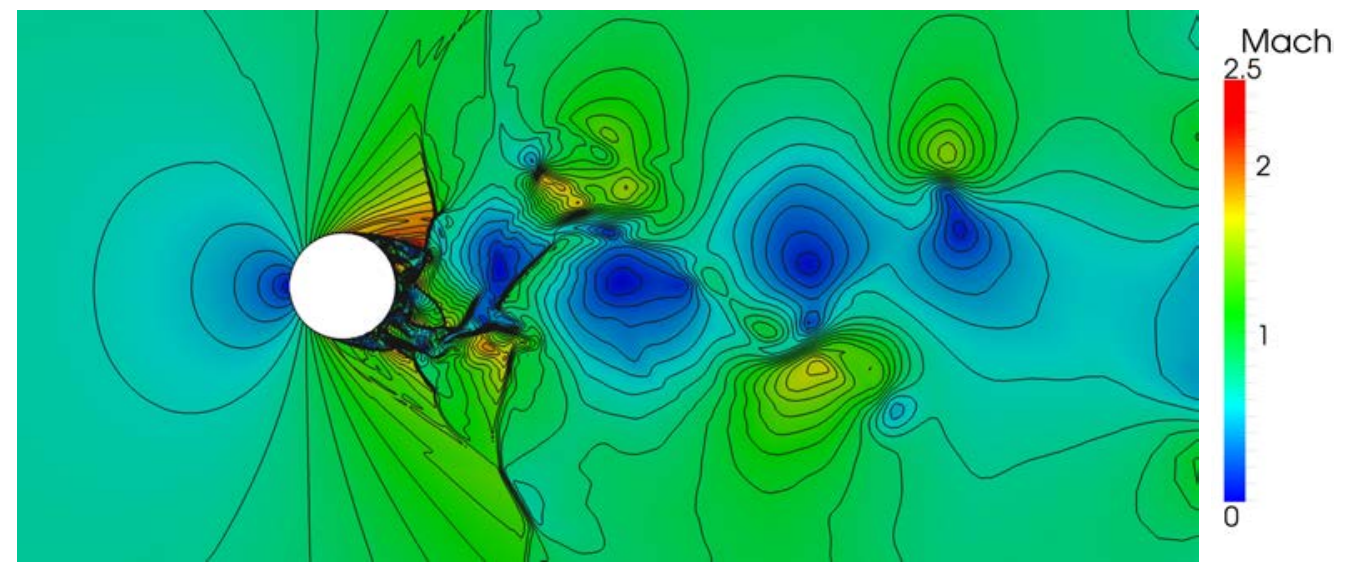

Figure 17: Unsteady transonic viscous flow over a circular cylinder. Mach number field for $t^{*}=49.93$.

The efficiency of the present shock-sensor strategy is highlighted in Figure 18. Due to the use of the MLS-based shock sensor, the Van Albada limiter are inactive on a great part of the low-mach region, thus preserving the accuracy of high order numerical scheme. 


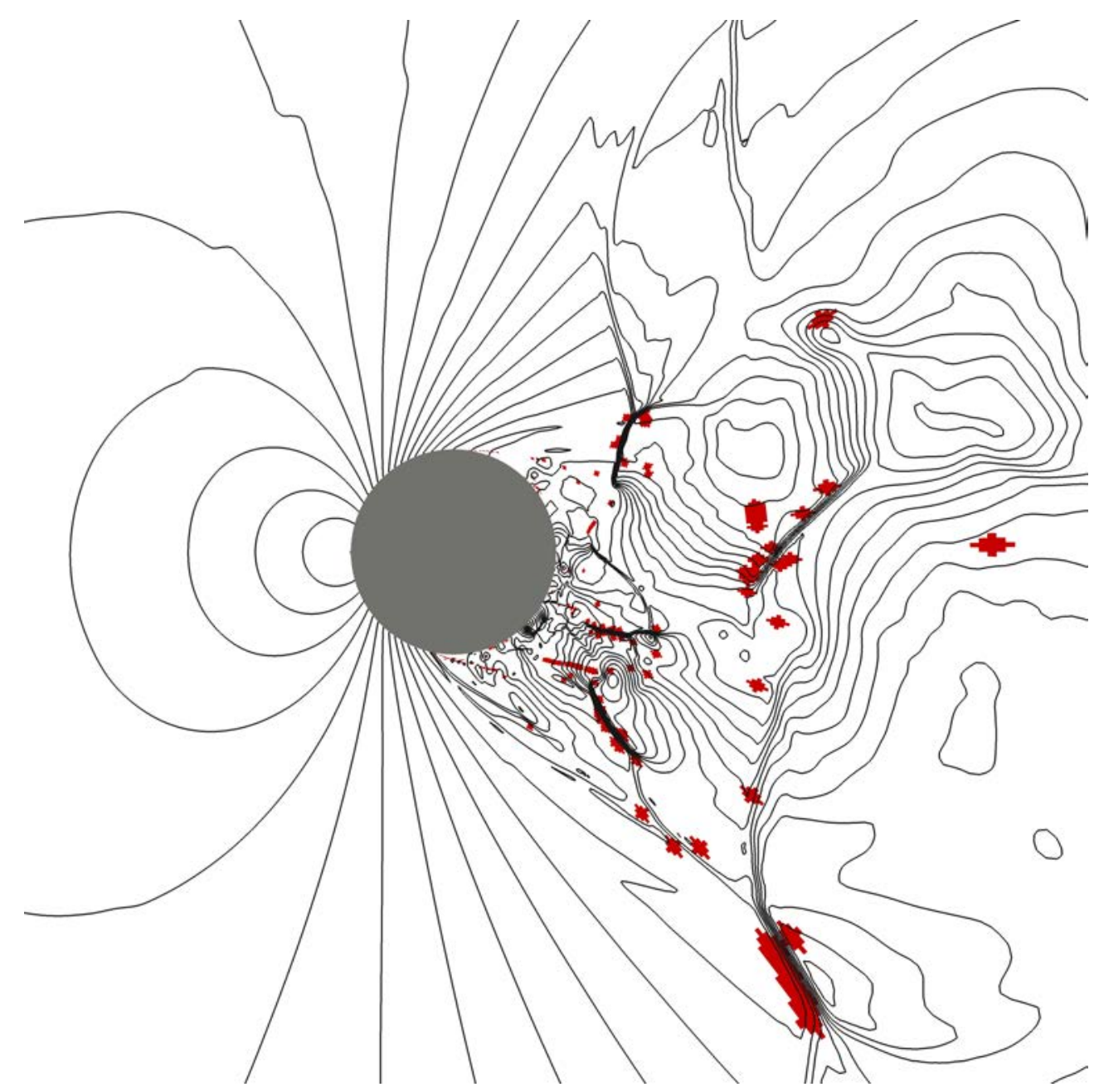

Figure 18: Unsteady transonic viscous flow over a circular cylinder. Pressure field and selective limiting for $t^{*}=49.93$. Shaded cells indicates the elements where the slope limiter is activated.

In order to investigate the performance of using low Mach fixes and MLS sensor, we now compute the time-averaged pressure coefficient $\overline{C_{p_{m}}}$ over the cylinder wall [56] 


$$
\overline{C_{p_{m}}}=\frac{(m-1){\overline{C_{p_{m-1}}}}+C_{p_{m}}}{m}
$$

where $m$ is the number of the averaged instants, and the pressure coefficient $C_{p_{m}}$ is obtained for each position $\theta$ as

$$
C_{p_{m}}(\theta)=\frac{p(\theta)-p_{\infty}}{\frac{1}{2} \rho_{\infty}\left|u_{\infty}\right|}
$$

The angle $\theta$ used for this definition is schematically shown in Figure 19.

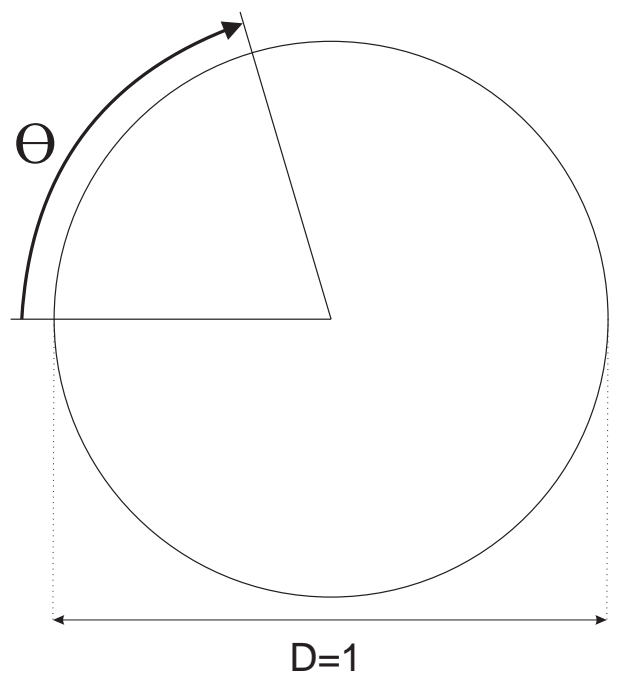

Figure 19: Definition of $\theta$ for the computation of $C_{p_{m}}$ over the cylinder wall.

Figure 20 presents a comparison of the distribution of $\overline{C_{p_{m}}}$ obtained for various numerical strategies. We remark that it is necessary to consider both the low Mach fix and the MLS sensor in order to obtain a correct mean position of the boundary layer separation point, situated at around 70 degrees [53]. On the contrary, computations without low Mach fix predicts a position of the boundary layer separation point around 60 degrees, which 
corresponds to a more dissipative solution. In addition, the use of the MLS sensor gives less dissipative results than those obtained using the Venkatakrishnan limiter. Minor discrepancies are visible for $\theta$ greater than 100 degrees between results obtained using the Rieper's fix or the tangential fix presented in Section 3.1.4.

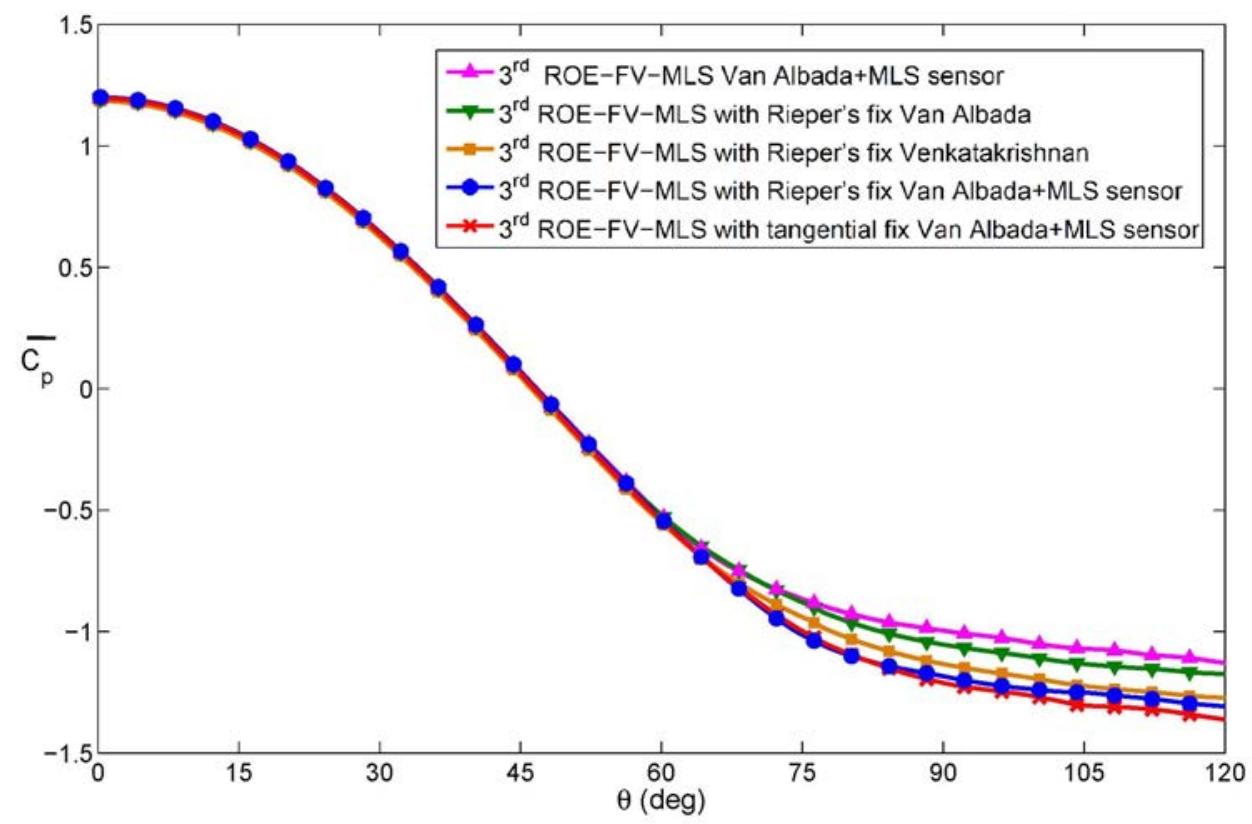

Figure 20: Unsteady transonic viscous flow over a circular cylinder.Time-averaged surface pressure coefficient around the cylinder.

\section{Conclusions}

In this work we have presented a high-order density-based finite volume framework for all-speed flows. The main ingredients of this formulation relies on a MLS-based finite volume formulation, a low-Mach fix and a slope 
limiting strategy coupled with a MLS-based shock sensor. We have shown that the accuracy problem in finite volume schemes can be alleviated simply using high-order discretization scheme. The problem of grid dependency of the solution accuracy with the Mach number was thoroughly investigated for both the fluxes of Roe and Rusanov. In particular, we have found that this dependence is smaller than for the first-order numerical scheme. Moreover we demonstrated that use of high-order schemes in conjunction with low-Mach fixes proposed by Rieper for the first-order Roe scheme and those proposed by $\mathrm{Li}$ and $\mathrm{Gu}$ for the Rusanov fluxes successfully preserves the accuracy of the solution at low Mach numbers. Finally the use of MLS-based shock wave sensor prevents unnecessarily activation of the slope limiter, thus avoiding the presence of spurious pressure oscillations in low-Mach regions.

\section{Acknowledgments}

This work has been partially supported by the Ministerio de Ciencia

e Innovación (grant \#DPI2010-16496) and the Ministerio de Economía y Competitividad (grant \#DPI2012-33622) of the Spanish Government and by the, Consellería de Cultura, Educación e Ordenación Universitaria of the Xunta de Galicia (grant \#GRC2014/039 ) cofinanced with FEDER funds, and the Universidade da Coruña.

\section{References}

[1] Roe, P. L., Approximate Riemann solvers, parameter vectors and difference schemes, Journal of Computational Physics,43, 357-372, 1981. 
[2] Van Leer, B., Flux vector splitting for the Euler equations, Lecture Notes in Physics, 170. Springer Verlag, 1982.

[3] Colella, P., Woodward, P., The piecewise parabolic method (PPM) for gas-dynamical simulations, Journal of Computational Physics, 54:174-201, 1984.

[4] A. J. Chorin, Numerical solution of the Navier-Stokes equations, Mathematics of Computation,22:745-762, 1968.

[5] S. V. Patankar, Numerical heat transfer and fluid flow, McGraw-Hill, New York, 1980.

[6] R. Peiret and T. D. Taylor, Computational Methods for fluid flow, Springer-Verlag, New York, 1983.

[7] K. Karki, S. V. Patankar, Pressure-based calculation procedure for viscous flows at all speeds in arbitrary configurations, AIAA Journal, 27(9):11671174, 1989.

[8] C. D. Munz, S. Roller, R. Klein, K. J. Geratz, The extension of incompressible flow solvers to the weakly compressible regime, Computers and Fluids, 32(2):173-196, 2003.

[9] S. Roller and C. D. Munz, A low Mach number scheme based on multiscale asymptotics, Computing and Visualization in Science, 3:85-91, 2000.

[10] J. Sesterhenn, B. Müller, H. Thomann, On the cancellation problem in calculating compressible low Mach number flows, Journal of Computational Physics, 151 (2):597-615, 1999. 
[11] S. Klainerman, A. Majda, Compressible and incompressible fluids, Communications on Pure and Applied Mathematics, 35:629-651, 1982.

[12] H. Guillard, C. Viozat, On the behaviour of upwind schemes in the low mach number limit, Computers and Fluids, 28:63-86, 1999.

[13] H. Guillard, A. Murrone, On the behaviour of upwind schemes in the low mach number limit: II. Godunov type schemes, Computers and Fluids, 33:655-675, 2004.

[14] S. Dellacherie, Analysis of Godunov type schemes applied to the compressible Euler system at low Mach number, Journal of Computational Physics, 229(4):978-1016, 2010.

[15] X.-S Li, C.-W Gu, An All-Speed Roe-type scheme and its asymptotic analysis of low Mach number behaviour, Journal of Computational Physics, 22\%:5144-5159, 2008.

[16] E. Turkel, Preconditioned methods for solving the incompressible and low speed compressible equations, Journal of Computational Physics, 72:277298, 1987.

[17] C. L. Merkle, Y.-H. Choi, Computation of low-speed compressible flows with time-marching procedures, International Journal for Numerical Methods in Engineering, 25(2):293-311, 1988.

[18] B. Van Leer, W.-T. Lee and P. L. Roe, Characteristic time-stepping or local preconditioning of the Euler equations,AIAA Computational Fluid Dynamics Conference, 10th, Honolulu, HI, June 24-27, 1991, Technical 
Papers (A91-40701 17-34). Washington, DC, American Institute of Aeronautics and Astronautics, 260-282, 1991.

[19] C. L. Merkle and M. Athavale, Time Accurate Unsteady Incompressible Flow Algorithm Based on Artificial Compressibility,AIAA 8th Computational Fluid Dynamics Conference, Honolulu, AIAA Paper 87-1137, 1987.

[20] S. Venkateswaran and C. L. Merkle, Dual time stepping and preconditioning for unsteady computations, 33rd Aerospace Sciences Meeting and Exhibit, Reno, Nevada, AIAA Paper 95-0078, 1995.

[21] F. Cordier, P. Degond, A. Kumbaro, An Asymptotic-Preserving all-speed scheme for the Euler and NavierStokes equations, Journal of Computational Physics, 231(17):5685-5704, 2012.

[22] H. Paillère, C. Viozat, A. Kumbaro, I. Toumi, Comparison of low Mach number models for natural convection problem, Heat Mass Transfer 36, 567573, 2000.

[23] P. Fillion, A. Chanoine, S. Dellacherie, A. Kumbaro, FLICA-OVAP: A new platform for core thermalhydraulic studies, Nuclear Engineering and Design 241, 4348-4358, 2011.

[24] B. Thornber, D. Drikakis, R.J.R Williams, D. Youngs, On entropy generation and dissipation of kinetic energy in high-resolution shock-capturing schemes, Journal of Computational Physics, 227:4853-4872, 2008.

[25] F. Rieper, G. Bader, The influence of cell geometry on the accuracy of upwind schemes in the low Mach number regime, Journal of Computational Physics, 228:2918-2933, 2009. 
[26] B. Thornber, D. Drikakis, Numerical dissipation of upwind schemes in low mach flow, International Journal for Numerical Methods in Fluids, $56: 1535-1541,2008$.

[27] F. Rieper, A low-Mach number fix for Roe's approximate Riemann solver, Journal of Computational Physics, 230:5263-5287, 2011.

[28] B. Thornber, A. Mosedale, D. Drikakis, D. Youngs, R.J.R Williams, An improved reconstruction method for compressible flows with low Mach number features, Journal of Computational Physics, 227:4873-4894, 2008.

[29] X.-S Li, C.-W Gu, Mechanism of Roe-type schemes for all-speed flows and its application, Computers and Fluids, 86:56-70, 2013.

[30] M.-S Liou, A sequel to AUSM, Part II: AUSM+ -up for all speeds, Journal of Computational Physics, 214:137-170, 2006.

[31] E. Shima and K. Kitamura, On New Simple Low-Dissipation Scheme of AUSM-Family for All Speeds, AIAA paper 2009-136, 2009.

[32] E. Shima, K. Kitamura, Performance of Low-Dissipation Euler Fluxes and Preconditioned Implicit Schemes in Low Speeds, Communications in Computational Physics, 10:90-119, 2011.

[33] H. Luo, J.D. Baum, R. Lohner A fast p-multigrid discontinuous Galerkin method for compressible flows at all speeds, in: 44th AIAA Aerospace Sciences Meeting and Exhibit, Reno, 2006.

[34] M. Feistauer, V. Kucera, On a robust discontinuous Galerkin technique 
for the solution of compressible flow, Journal of Computational Physics, 224(1):208-221, 2007.

[35] F. Bassi, C. De Bartolo, R. Hartmann, A. Nigro, A discontinuous Galerkin method for inviscid low Mach number flows, Journal of Computational Physics, 228:3996-4011, 2009.

[36] L. Cueto-Felgueroso, I. Colominas, X. Nogueira, F. Navarrina, M. Casteleiro, Finite volume solvers and Moving Least-Squares approximations for the compressible Navier-Stokes equations on unstructured grids, Computer Methods in Applied Mechanics and Engineering, 196:4712-4736, 2007.

[37] X. Nogueira, L. Cueto-Felgueroso, I. Colominas, H. Gómez, F. Navarrina, M. Casteleiro, On the accuracy of Finite Volume and Discontinuous Galerkin discretizations for compressible flow on unstructured grids, International Journal for Numerical Methods in Engineering, 78:1553-1584, 2009.

[38] X. Nogueira, L. Cueto-Felgueroso, I. Colominas, S. Khelladi, On the simulation of wave propagation with a higher-order finite volume scheme based on Reproducing Kernel Methods, Computer Methods in Applied Mechanics and Engineering, 199(23-24):1471-1490, 2010.

[39] S. Khelladi, X. Nogueira, F. Bakir, I. Colominas, Toward a Higher Order Unsteady Finite Volume Solver Based on Reproducing Kernel Methods, Computer Methods in Applied Mechanics and Engineering, 200:2348-2362, 2011. 
[40] L. Ramirez, X. Nogueira, S. Khelladi, J.C. Chassaing, and I. Colominas, A new higher-order finite volume method based on Moving Least Squares for the resolution of the incompressible Navier-Stokes equations on unstructured grids. Computer Methods in Applied Mechanics and Engineering, 278, 883-901, 2014.

[41] P. Lancaster, K. Salkauskas, Surfaces generated by moving least squares methods, Mathematics of Computation, 37(155), 141-178, 1981.

[42] W. K. Liu, W. Hao, Y. Chen, S. Jun, J. Gosz, Multiresolution Reproducing Kernel Particle Methods, Computational Mechanics, 20, 295-309, 1997.

[43] S. Schochet, Fast singular limits of hyperbolic PDEs, Journal of Differential Equations 114:476-512, 1994.

[44] C.-W. Shu, S. Osher, Efficient implementation of essentially nonoscillatory shock-capturing schemes, Journal of Computational Physics, 77, 439471, 1998.

[45] V. V. Rusanov, The calculation of the interaction of non-stationary shock waves and obstacles, USSR Computational Mathematics and Mathematical Physics, 1(2):304-320, 1962.

[46] F. Rieper, On the dissipation mechanism of upwind-schemes in the low Mach number regime: A comparison between Roe and HLL, Journal of Computational Physics, 229:221-232, 2010.

[47] G. D. Van Albada, B. Van Leer, W. W. Roberts, A Comparative study 
of computational methods in cosmic gas dynamics, Astronomy and Astrophysics, 108, 76-84, 1982.

[48] T. J.Barth, D. C. Jespersen, The design and application of upwind schemes on unstructured meshes, AIAA-89-0366, 1989.

[49] V. Venkatakrishnan, Convergence to steady state solutions of the Euler equations on unstructured grids with limiters, Journal of Computational Physics, 118, 120-130, 1995.

[50] X. Nogueira, L. Cueto-Felgueroso, I. Colominas, F. Navarrina, M. Casteleiro, A new shock-capturing technique based on Moving Least Squares for higher-order numerical schemes on unstructured grids, Computer Methods in Applied Mechanics and Engineering, 199: 2544-2558, 2010.

[51] S. J. Schmidt, I. H. Sezal, G. H. Schnerr and M. Talhamer Riemann techniques for the simulation of compressible liquid flow with phase-transition at all Mach numbers - Shock and wave dynamics in cavitating 3D Mmicro and macro systems, AIAA paper 2008-1238,in: 46th AIAA Aerospace Sciences Meeting and Exhibit, Reno, 2006.

[52] F. Rieper, On the Behaviour of Numerical Schemes in the Low Mach Number Regime, PhD. Thesis, Brandenburgischen Technischen Universität Cottbus, 2008.

[53] V.S. Murthy, W. C. Rose, Detailed Measurements on a Circular Cylinder in Cross Flow, AIAA Journal,57, 549550, 1978.

[54] J.P. Boris, F.F. Grinstein, E.S. Oran, R.S. Kolbe, New insights into large eddy simulation, Fluid Dynamics Research,10, 199-228, 1992. 
[55] X. Nogueira, L. Cueto-Felgueroso, I. Colominas, H. Gomez, Implicit Large Eddy Simulation of non-wall-bounded turbulent flows based on the multiscale properties of a high-order finite volume method, Computer Methods in Applied Mechanics and Engineering, 199:615624, 2010.

[56] P. Majander, T. Siikonen, Evaluation of Smagorinsky-based subgrid-scale models in a finite-volume computation, International Journal for Numerical Methods in Fluids, 40:735-774, 2002. 